\title{
Quantifying The Complexity Of Geodesic Paths On Curved Statistical Manifolds Through Information Geometric Entropies and Jacobi Fields
}

\author{
Carlo Cafard* and Stefano Mancin用 \\ Dipartimento di Fisica, Università di Camerino, I-62032 Camerino, Italy
}

\begin{abstract}
We characterize the complexity of geodesic paths on a curved statistical manifold $\mathcal{M}_{s}$ through the asymptotic computation of the information geometric complexity $\mathcal{V}_{\mathcal{M}_{s}}$ and the Jacobi vector field intensity $J_{\mathcal{M}_{s}}$. The manifold $\mathcal{M}_{s}$ is a $2 l$-dimensional Gaussian model reproduced by an appropriate embedding in a larger $4 l$-dimensional Gaussian manifold and endowed with a Fisher-Rao information metric $g_{\mu \nu}(\Theta)$ with non-trivial off diagonal terms. These terms emerge due to the presence of a correlational structure (embedding constraints) among the statistical variables on the larger manifold and are characterized by macroscopic correlational coefficients $r_{k}$. First, we observe a power law decay of the information geometric complexity at a rate determined by the coefficients $r_{k}$ and conclude that the non-trivial off diagonal terms lead to the emergence of an asymptotic information geometric compression of the explored macrostates $\Theta$ on $\mathcal{M}_{s}$. Finally, we observe that the presence of such embedding constraints leads to an attenuation of the asymptotic exponential divergence of the Jacobi vector field intensity.
\end{abstract}

PACS numbers: Probability Theory (02.50.Cw), Riemannian Geometry (02.40.Ky), Chaos (05.45.-a), Complexity (89.70.Eg), Entropy (89.70.Cf).

\section{INTRODUCTION}

Characterizing and understanding the mystery of the origin of life and the unfolding of its evolution are perhaps the leading arguments motivating the quantification of the extremely elusive concept of complexity [1-3]. Of course there are more pragmatic reasons that justify the study of complexity, for example the problem of quantifying how complex is quantum motion. This issue is of primary importance in quantum information science. However, our knowledge of the relations between complexity, dynamical stability, and chaoticity in a fully quantum domain is still not satisfactory [4, 5]. The concept of complexity is very difficult to define, its origin is not fully understood [6 11] and it is mainly for this reason that several quantitative measures of complexity have appeared in the scientific literature [1-3]. In classical physics, measures of complexity are settled in a better way. The Kolmogorov-Sinai metric entropy [12], that is the sum of all positive Lyapunov exponents [13], is a powerful indicator of unpredictability in classical systems and it measures the algorithmic complexity of classical trajectories [14 17]. Other known measures of complexity are the logical depth [18], the thermodynamic depth [19], the computational complexity [20], the stochastic complexity [21] and many more. Ideally, a good definition of complexity should be mathematically rigorous and intuitive at the same time so that it allows to tackle complexity-related problems in computation theory and statistical physics as well. Of course, a quantitative measure of complexity is truly useful if its range of applicability is not limited to few unrealistic applications. It is also for this reason that in order to properly define complexity measures, it should be clearly stated the reasons why one is defining such a measure and what it is intended to capture.

It is known that classical complex systems exhibit local exponential instability and are characterized by positive Lyapunov exponents [22]. Furthermore, the study of the relationship between entropy and the complexity of trajectories of a dynamical system has always been an active field of research [14 16]. In particular, in [14] it was shown that the algorithmic complexity of trajectories of points in a dynamical system is asymptotically equal to the entropy of the system.

It is commonly accepted that one of the major goals of physics is modeling and predicting natural phenomena using relevant information about the system of interest. Taking this statement seriously, it is reasonable to expect that the laws of physics should reflect the methods for manipulating information. Indeed, the less controversial opposite point of view may be considered where the laws of physics are used to manipulate information. This is exactly the point of view adopted in quantum information science where information is manipulated using the laws of quantum mechanics [23].

Here we make use of the so-called Entropic Dynamics (ED) 24] and Information Geometrodynamical Approach

\footnotetext{
*Electronic address: carlo.cafaro@unicam.it

${ }^{\dagger}$ Electronic address: stefano.mancini@unicam.it
} 
to Chaos (IGAC) 25, 26]. ED is a theoretical framework that arises from the combination of inductive inference (Maximum Entropy Methods, 27 30]) and Information Geometry [31]. The most intriguing question being pursued in ED stems from the possibility of deriving dynamics from purely entropic arguments. This is clearly valuable in circumstances where microscopic dynamics may be too far removed from the phenomena of interest, such as in complex biological or ecological systems, or where it may just be unknown or perhaps even nonexistent, as in economics. The applicability of ED has been extended to temporally-complex (chaotic) dynamical systems on curved statistical manifolds and relevant measures of chaoticity of such an information geometrodynamical approach to chaos have been identified [25]. IGAC arises as a theoretical framework to study chaos in informational geodesic flows describing physical, biological or chemical systems. A geodesic on a curved statistical manifold $\mathcal{M}_{S}$ represents the maximum probability path a complex dynamical system explores in its evolution between initial and final macrostates. Each point of the geodesic is parametrized by the macroscopic dynamical variables $\{\Theta\}$ defining the macrostate of the system. Furthermore, each macrostate is in a one-to-one correspondence with the probability distribution $\{p(X \mid \Theta)\}$ representing the maximally probable description of the system being considered. The quantity $X$ is a microstate of the microspace $\mathcal{X}$. The set of macrostates forms the parameter space $\mathcal{D}_{\Theta}$ while the set of probability distributions forms the statistical manifold $\mathcal{M}_{S}$. IGAC is the information geometric analogue of conventional geometrodynamical approaches [32, 33] where the classical configuration space $\Gamma_{E}$ is being replaced by a statistical manifold $\mathcal{M}_{S}$ with the additional possibility of considering chaotic dynamics arising from non conformally flat metrics (the Jacobi metric is always conformally flat, instead). It is an information geometric extension of the Jacobi geometrodynamics (the geometrization of a Hamiltonian system by transforming it to a geodesic flow [34]). The reformulation of dynamics in terms of a geodesic problem allows the application of a wide range of well-known geometrical techniques in the investigation of the solution space and properties of the equation of motion. The power of the Jacobi reformulation is that all of the dynamical information is collected into a single geometric object in which all the available manifest symmetries are retained- the manifold on which geodesic flow is induced. For example, integrability of the system is connected with existence of Killing vectors and tensors on this manifold. The sensitive dependence of trajectories on initial conditions, which is a key ingredient of chaos, can be investigated from the equation of geodesic deviation. In the Riemannian [32] and Finslerian [33] (a Finsler metric is obtained from a Riemannian metric by relaxing the requirement that the metric be quadratic on each tangent space) geometrodynamical approach to chaos in classical Hamiltonian systems, an active field of research concerns the possibility of finding a rigorous relation among the sectional curvature, the Lyapunov exponents, and the Kolmogorov-Sinai dynamical entropy [35].

In this article, inspired by the work presented in [36], using statistical inference and information geometric techniques, we characterize the complexity of geodesic paths on a curved statistical manifold $M_{s}$ through the asymptotic computation of the information geometric complexity $\mathcal{V}_{\mathcal{M}_{s}}$ and the Jacobi vector field $J_{\mathcal{M}_{s}}$. The manifold $\mathcal{M}_{s}$ is a $2 l$-dimensional Gaussian model reproduced by an appropriate embedding in a larger $4 l$-dimensional Gaussian manifold and endowed with a Fisher-Rao information metric $g_{\mu \nu}(\Theta)$ with non-trivial off diagonal terms. These terms in the information metric on the embedded manifold emerge due to the presence of a correlational structure (embedding constraints) among the statistical variables on the larger manifold and are characterized by macroscopic correlational coefficients $r_{k}$. First, we observe a power law decay of the information geometric complexity $\mathcal{V}_{\mathcal{M}_{s}}(\tau) \approx \exp \left[\mathcal{S}_{\mathcal{M}_{s}}(\tau)\right]$ at a rate determined by the coefficients $r_{k}$ and conclude that the non-trivial off diagonal terms lead to the emergence of an asymptotic information geometric compression of the explored macrostates $\Theta$ on $\mathcal{M}_{s}$. Finally, we observe that the presence of such embedding constraints leads to an attenuation of the asymptotic exponential divergence of the Jacobi vector field intensity.

The layout of this article is as follows. In Section II, we present few remarks on the theoretical structure of the IGAC and outline few selected applications concerning the complexity characterization of geodesic paths on curved statistical manifolds. In Section III, we describe the $2 l$-dimensional curved statistical model considered, the embedded Gaussian model endowed with a Fisher-Rao information metric with non-trivial off diagonal terms. In Section IV, we present the asymptotic computation of the information geometric entropy $\mathcal{S}_{\mathcal{M}_{s}}(\tau)$. We observe a power law decay of the information geometric complexity $\mathcal{V}_{\mathcal{M}_{s}}(\tau)$ at a rate determined by the coefficients $r_{k}$ and conclude that nontrivial off diagonal terms lead to the emergence of an asymptotic information geometric compression of the explored macrostates on the statistical configuration manifold considered. In Section V, we present the asymptotic computation of the Jacobi fields on $\mathcal{M}_{S}$. We observe that the presence of the embedding constraints lead to an attenuation of the asymptotic exponential divergence of the Jacobi field intensity. Finally, in Section VI we present final remarks and suggest further research directions.

\section{ON THE IGAC: REMARKS AND APPLICATIONS}

In this Section, we present few remarks on the theoretical structure of the IGAC and outline few selected applications concerning the complexity characterization of geodesic paths on curved statistical manifolds. A more detailed review 
appears in [25].

\section{A. Remarks}

As stated in the Introduction, the IGAC arises as an information geometric framework to study chaos and complexity in informational geodesic flows describing physical systems. A geodesic on a curved statistical manifold represents the maximum probability path a complex dynamical system explores in its evolution between initial and final macrostates $\Theta_{i}$ and $\Theta_{f}$, respectively. Each point of the geodesic on a $4 l$-dimensional statistical manifold represents a macrostate $\Theta$ parametrized by the macroscopic dynamical variables $\Theta \equiv\left(\theta_{1}, \ldots, \theta_{4 l}\right)$ defining the macrostate of the system. Furthermore, each macrostate is in a one-to-one correspondence with the probability distribution $P(X \mid \Theta)$ representing the maximally probable description of the system being considered. The set of macrostates forms the parameter space while the set of probability distributions form the statistical manifold. In what follows, we schematically outline the main features underlying the construction of an arbitrary form of entropic dynamics. First, the microstates of the system under investigation must be defined. For the sake of reasoning, we assume the system is characterized by an $2 l$-dimensional microspace with microstates $X \equiv\left(x_{1}, \ldots, x_{2 l}\right)$. The main goal of an ED model is that of inferring "macroscopic predictions" in the absence of detailed knowledge of the microscopic nature of the arbitrary complex systems being considered. More explicitly, by "macroscopic prediction" we mean knowledge of the statistical parameters (expectation values) of the probability distribution function that best reflects what is known about the system. This is an important conceptual point. The probability distribution reflects the system in general, not the microstates. Once the microstates have been defined, we then select the relevant information about the system. In other words, we have to select the macrospace of the system.

In general, one is given a manifold of probability distributions arising from the maximum entropy formalism where distributions arise from the maximization of the logarithmic relative entropy subjected to some statements concerning averages (information constraints). Given the manifold of probability distributions, the (direct) problem is to find the corresponding Fisher-Rao information metric. However, not all probability distributions are generated in this way. For instance, probability distributions may emerge as a result of a change of variable technique (parametric transformation law) [37]. Furthermore, in order to do physics, we are also concerned with the following (inverse) problem: we want to design statistical manifolds with appropriate geometries [38].

\section{B. Applications}

In the following, we outline few selected applications concerning the complexity characterization of geodesic paths on curved statistical manifolds.

\section{Gaussian Statistical Models in the Absence of Correlations}

In [36], we apply the IGAC to study the dynamics of a system with $l$ degrees of freedom, each one described by two pieces of relevant information, its mean expected value and its variance (Gaussian statistical macrostates). This leads to consider a statistical model on a non-maximally symmetric $2 l$-dimensional statistical manifold $\mathcal{M}_{s}$. It is shown that $\mathcal{M}_{s}$ possesses a constant negative scalar curvature proportional to the number of degrees of freedom of the system, $\mathcal{R}_{\mathcal{M}_{s}}=-l$. It is found that the system explores statistical volume elements on $\mathcal{M}_{s}$ at an exponential rate. The information geometric entropy $\mathcal{S}_{\mathcal{M}_{s}}$ increases linearly in time (statistical evolution parameter) and, moreover, is proportional to the number of degrees of freedom of the system, $\mathcal{S}_{\mathcal{M}_{s}} \stackrel{\tau \rightarrow \infty}{\sim} l \lambda \tau$ where $\lambda$ is the maximum positive Lyapunov exponent characterizing the model. The asymptotic linear information geometric entropy growth may be considered an information-geometric analogue of the von Neumann entropy growth introduced by Zurek-Paz, a quantum feature of chaos. The geodesics on $\mathcal{M}_{s}$ are hyperbolic trajectories. Using the Jacobi-Levi-Civita (JLC) equation for geodesic spread, we show that the Jacobi vector field intensity $J_{\mathcal{M}_{s}}$ diverges exponentially and is proportional to the number of degrees of freedom of the system, $J_{\mathcal{M}_{s}} \stackrel{\tau \rightarrow \infty}{\sim} l \exp (\lambda \tau)$. The exponential divergence of the Jacobi vector field intensity $J_{\mathcal{M}_{s}}$ is a classical feature of chaos. Therefore, we conclude that $\mathcal{R}_{\mathcal{M}_{s}}=-l, J_{\mathcal{M}_{s}} \stackrel{\tau \rightarrow \infty}{\sim} l \exp (\lambda \tau)$ and $\mathcal{S}_{\mathcal{M}_{s}} \stackrel{\tau \rightarrow \infty}{\sim} l \lambda \tau$. Thus, $\mathcal{R}_{\mathcal{M}_{s}}, \mathcal{S}_{\mathcal{M}_{s}}$ and $J_{\mathcal{M}_{s}}$ behave as proper indicators of chaoticity and are proportional to the number of Gaussian-distributed microstates of the system. This proportionality, even though proven in a very special case, leads to conclude there may be a substantial link among these information geometric indicators of chaoticity. 


\section{Gaussian Statistical Models in the Presence of Correlations between Microvariables}

In [39], we apply the IGAC to study the information constrained dynamics of a system with $l=2$ microscopic degrees of freedom. As working hypothesis, we assume that such degrees of freedom are represented by two correlated Gaussian-distributed microvariables characterized by the same variance. We show that the presence of microcorrelations lead to the emergence of an asymptotic information geometric compression of the statistical macrostates explored by the system at a faster rate than that observed in absence of microcorrelations. This result constitutes an important and explicit connection between (micro)-correlations and (macro)-complexity in statistical dynamical systems. The relevance of our finding is twofold: first, it provides a neat description of the effect of information encoded in microscopic variables on experimentally observable quantities defined in terms of dynamical macroscopic variables; second, it clearly shows the change in behavior of the macroscopic complexity of a statistical model caused by the existence of correlations at the underlying microscopic level.

\section{Ensemble of Random Frequency Macroscopic Inverted Harmonic Oscillators}

In [38], we explore the possibility of using well established principles of inference to derive Newtonian dynamics from relevant prior information codified into an appropriate statistical manifold. The basic assumption is that there is an irreducible uncertainty in the location of particles so that the state of a particle is defined by a probability distribution. The corresponding configuration space is a statistical manifold the geometry of which is defined by the Fisher-Rao information metric. The trajectory follows from a principle of inference, the method of Maximum Entropy. There is no need for additional "physical" postulates such as an action principle or equation of motion, nor for the concept of mass, momentum and of phase space, not even the notion of time. The resulting "entropic" dynamics reproduces Newton's mechanics for any number of particles interacting among themselves and with external fields. Both the mass of the particles and their interactions are explained as a consequence of the underlying statistical manifold.

Following this line of reasoning, in 26, 40] we present an information geometric analogue of the Zurek-Paz quantum chaos criterion in the classical reversible limit. This analogy is illustrated by applying the IGAC to a set of $n$-uncoupled three-dimensional anisotropic inverted harmonic oscillators characterized by a Ohmic distributed frequency spectrum.

\section{IGAC of regular and chaotic quantum spin chains}

In [41, 42], we study the entropic dynamics on curved statistical manifolds induced by classical probability distributions of common use in the study of regular and chaotic quantum energy level statistics. Specifically, we propose an information geometric characterization of chaotic (integrable) energy level statistics of a quantum antiferromagnetic Ising spin chain in a tilted (transverse) external magnetic field. We consider the IGAC of a Poisson distribution coupled to an Exponential bath (spin chain in a transverse magnetic field, regular case) and that of a Wigner-Dyson distribution coupled to a Gaussian bath (spin chain in a tilted magnetic field, chaotic case). Remarkably, we show that in the former case the IGE exhibits asymptotic logarithmic growth while in the latter case the IGE exhibits asymptotic linear growth.

\section{THE MODEL}

In this Section, we emphasize the main reasoning underlying the choice of the new proposed statistical model and study its information geometric properties.

\section{A. Motivations}

We want to make reliable macroscopic predictions when only partial knowledge on the micro-structure of a system is available. As stated in the Introduction, the complexity of such predictions is quantified in terms of the IGE and the Jacobi field intensity. In this manuscript we seek an answer to the following question: isn't simpler to make macroscopic predictions when the available pieces of information are not independent? Stated otherwise, does an increase in the correlational structure of the dynamical equations for the statistical variables labelling a macrostate of a system imply a reduction in the complexity of the geodesic paths? It is reasonable to expect that the emergence of 
a correlational structure in the form of constraints among the variables labelling the macrostates of a system would lead to a highly constrained dynamics and, consequently, to a reduction in the complexity of making macroscopic predictions. In what follows, we attempt to give a quantitative answer to the above-mentioned questions.

We propose to compare the complexity of making predictions in two different scenarios. In the first scenario (4ldimensional larger Gaussian model), we assume a system with $2 l$ degrees of freedom $x_{k}$, each one being Gaussian distributed. The probability distribution describing the whole system is given by,

$$
P(X \mid \Theta)=\prod_{k=1}^{2 l} p\left(x_{k} \mid \mu_{k}, \sigma_{k}\right)
$$

where $X \equiv\left(x_{1}, \ldots, x_{2 l}\right), \Theta \equiv\left(\mu_{1}, \ldots, \mu_{2 l}, \sigma_{1}, \ldots, \sigma_{2 l}\right)$ and $p\left(x_{k} \mid \mu_{k}, \sigma_{k}\right)$ is defined as,

$$
p\left(x_{k} \mid \mu_{k}, \sigma_{k}\right) \stackrel{\text { def }}{=} \frac{1}{\sqrt{2 \pi \sigma_{k}^{2}}} \exp \left[-\frac{\left(x_{k}-\mu_{k}\right)^{2}}{2 \sigma_{k}^{2}}\right] .
$$

In [36, 44], we provided an analytical estimate for the complexity of geodesic paths on the statistical manifolds of distributions given in (1). In the second scenario (2l-dimensional embedded Gaussian model), we consider the very same system with $2 l$ degrees of freedom $x_{k}$. However, the microvariables $x_{k}$ are described by probability distributions characterized by statistical variables subject to a set of $2 l$-constraints,

$$
\sigma_{2 j}=\sigma_{2 j-1} \text { and, } \mu_{2 j}=\mu_{2 j}\left(\mu_{2 j-1}, \sigma_{2 j-1}\right) \text { (embedding constraints), }
$$

with $j=1, \ldots, l$. Therefore, the probability distribution describing the whole system in this second scenario becomes,

$$
\tilde{P}(X \mid \tilde{\Theta})=\prod_{j=1}^{l} \tilde{p}\left(x_{2 j-1}, x_{2 j} \mid \mu_{2 j-1}, \sigma_{2 j-1}\right)
$$

where $X \equiv\left(x_{1}, \ldots, x_{2 l}\right), \tilde{\Theta} \equiv\left(\mu_{1}, \mu_{3}, \ldots, \mu_{2 l-1}, \sigma_{1}, \sigma_{3}, \ldots, \sigma_{2 l-1}\right)$ and $\tilde{p}\left(x_{2 j-1}, x_{2 j} \mid \mu_{2 j-1}, \sigma_{2 j-1}\right)$ is defined as,

$$
\tilde{p}\left(x_{2 j-1}, x_{2 j} \mid \mu_{2 j-1}, \sigma_{2 j-1}\right) \stackrel{\text { def }}{=} \frac{1}{2 \pi \sigma_{2 j-1}^{2}} \exp \left[-\frac{\left(x_{2 j-1}-\mu_{2 j-1}\right)^{2}+\left[x_{2 j}-\mu_{2 j}\left(\mu_{2 j-1}, \sigma_{2 j-1}\right)\right]^{2}}{2 \sigma_{2 j-1}^{2}}\right],
$$

with $j=1, \ldots, l$. Our purpose is computing the complexity of geodesic paths on the $2 l$-dimensional statistical manifold $\mathcal{M}_{S}^{(\text {embedded })} \equiv \mathcal{M}_{S}=\{\tilde{P}(X \mid \tilde{\Theta})\}$ and compare it with the one obtained on the $4 l$-dimensional manifold $\mathcal{M}_{S}^{\text {(larger) }}=\{P(X \mid \Theta)\}$. We expect that the emergence of the correlational structure defined in (3) between pairs of macroscopic statistical variables will give rise to a reduction of the system's complexity.

Except for an overall scale constant and a convenient re-scaling of variables, the information metric on the $2 l$ dimensional embedded manifold $\mathcal{M}_{S}$ is given by (see Appendix A for the explicit derivation),

$$
d S_{\mathcal{M}_{S}}^{2}=\sum_{j=1}^{l} \frac{1}{\sigma_{2 j-1}^{2}}\left(d \mu_{2 j-1}^{2}+2 r_{2 j-1} d \mu_{2 j-1} d \sigma_{2 j-1}+2 d \sigma_{2 j-1}^{2}\right)
$$

where the coefficients $r_{2 j-1}$ are defined as,

$$
r_{2 j-1} \stackrel{\text { def }}{=} \frac{\frac{\partial \mu_{2 j}}{\partial \mu_{2 j-1}} \frac{\partial \mu_{2 j}}{\partial \sigma_{2 j-1}}}{\left[1+\left(\frac{\partial \mu_{2 j}}{\partial \mu_{2 j-1}}\right)^{2}\right]^{\frac{1}{2}}\left[2+\frac{1}{2}\left(\frac{\partial \mu_{2 j}}{\partial \sigma_{2 j-1}}\right)^{2}\right]^{\frac{1}{2}}}
$$

The explicit expressions of such coefficients depend on the functional parametric form given to the embedding constraints $\mu_{2 j}=\mu_{2 j}\left(\mu_{2 j-1}, \sigma_{2 j-1}\right)$. More details are given in Appendix A. From (7) it follows that the coefficients $r_{2 j-1}$ are non-zero if and only if $\mu_{2 j}$ depends on both $\mu_{2 j-1}$ and $\sigma_{2 j-1}$. Therefore, we may conclude that the emergence of the non-trivial off-diagonal terms in (6) is a consequence of the previously mentioned correlational structure arising from the embedding constraints. Motivated by these considerations, we will name from now on the coefficients $r_{2 j-1}$, macroscopic correlational coefficients. 


\section{B. Information Geometry of the Model}

In this Subsection, we discuss the main steps leading to computation of the asymptotic temporal behavior of the dynamical complexity of geodesic trajectories for the $2 l$-dimensional Gaussian statistical model. For the sake of notational simplicity and in view of the involved analysis that we will present shortly, we replace $\tilde{\Theta}$ in (4) with $\Theta \equiv\left(\mu_{1}, \ldots, \mu_{l}, \sigma_{1}, \ldots, \sigma_{l}\right)$ and $\tilde{P}(X \mid \tilde{\Theta})$ in (4) with $P(X \mid \Theta)$ so that $\mathcal{M}_{S}^{(\text {embedded })} \equiv \mathcal{M}_{S}=\{P(X \mid \Theta)\}$. We begin to study the IGAC arising from the Fisher-Rao metric defined as,

$$
d s_{\mathcal{M}_{s}}^{2} \stackrel{\text { def }}{=} g_{i j}(\Theta) d \Theta^{i} d \Theta^{j}=\sum_{k=1}^{l}\left(\frac{1}{\sigma_{k}^{2}} d \mu_{k}^{2}+\frac{2 r_{k}}{\sigma_{k}^{2}} d \mu_{k} d \sigma_{k}+\frac{2}{\sigma_{k}^{2}} d \sigma_{k}^{2}\right) \text {, with } i, j=1, \ldots, 2 l .
$$

We assume positive macroscopic correlational coefficients $r_{k} \in(0,1), \forall k=1, \ldots, l$. The Fisher-Rao metric tensor $g_{i j}(\Theta) \stackrel{\text { def }}{=} g_{i j}\left(\mu_{1}, \ldots \mu_{l} ; \sigma_{1}, \ldots, \sigma_{l}\right)$ leading to the line element in (8) is given by,

$$
\left[g_{i j}(\Theta)\right]_{2 l \times 2 l}=\left(\begin{array}{cccc}
M_{2 \times 2}^{(1)} & 0 & 0 & 0 \\
0 & \cdot & 0 & 0 \\
0 & 0 & \cdot & 0 \\
0 & 0 & 0 & M_{2 \times 2}^{(l)}
\end{array}\right) \text {, with } i, j=1, \ldots, 2 l .
$$

where $M_{2 \times 2}^{(k)}$ is the two-dimensional matrix defined as,

$$
\left[M_{2 \times 2}^{(k)}\right] \stackrel{\text { def }}{=} \frac{1}{\sigma_{k}^{2}}\left(\begin{array}{cc}
1 & r_{k} \\
r_{k} & 2
\end{array}\right) \text { with } k=1, \ldots, l .
$$

The inverse matrix $\left[M_{2 \times 2}^{(k)}\right]^{-1}$, useful for computing the Christoffel connection coefficients and other quantities characterizing the information geometry of $\mathcal{M}_{s}$ is given by,

$$
\left[M_{2 \times 2}^{(k)}\right]^{-1} \stackrel{\text { def }}{=} \frac{\sigma_{k}^{2}}{2-r_{k}^{2}}\left(\begin{array}{cc}
2 & -r_{k} \\
-r_{k} & 1
\end{array}\right) \text { with } k=1, \ldots, l .
$$

It can be shown [43] that the scalar curvature of such $2 l$-dimensional manifold is given by,

$$
\mathcal{R}_{\mathcal{M}_{s}}\left(r_{1}, \ldots, r_{l}\right)=-2 \sum_{k=1}^{l}\left(2-r_{k}^{2}\right)^{-1} .
$$

Notice that in the limit of vanishing coefficients $\left\{r_{k}=0\right\}, \mathcal{R}_{\mathcal{M}_{s}}=-l$ as shown in [44]. The computation of geodesic equations on the $2 l$-dimensional Gaussian statistical manifold $\mathcal{M}_{s}$ leads to the following coupled systems of nonlinear second order ordinary differential equations,

$$
\begin{aligned}
& 0=\frac{d^{2} \mu_{k}}{d \tau^{2}}-\frac{r_{k}}{2-r_{k}^{2}} \frac{1}{\sigma_{k}}\left(\frac{d \mu_{k}}{d \tau}\right)^{2}-\frac{4}{2-r_{k}^{2}} \frac{1}{\sigma_{k}} \frac{d \mu_{k}}{d \tau} \frac{d \sigma_{k}}{d \tau}-\frac{2 r_{k}}{2-r_{k}^{2}} \frac{1}{\sigma_{k}}\left(\frac{d \sigma_{k}}{d \tau}\right)^{2}, \\
& 0=\frac{d^{2} \sigma_{k}}{d \tau^{2}}+\frac{1}{2-r_{k}^{2}} \frac{1}{\sigma_{k}}\left(\frac{d \mu_{k}}{d \tau}\right)^{2}+\frac{2 r_{k}}{2-r_{k}^{2}} \frac{1}{\sigma_{k}} \frac{d \mu_{k}}{d \tau} \frac{d \sigma_{k}}{d \tau}+\frac{2 r_{k}^{2}-2}{2-r_{k}^{2}} \frac{1}{\sigma_{k}}\left(\frac{d \sigma_{k}}{d \tau}\right)^{2} .
\end{aligned}
$$

with $k=1, \ldots, l$. When $r_{k} \rightarrow 0, \forall k$ we get the ordinary Gaussian system of nonlinear and coupled ordinary differential equations. Integration of such coupled system of nonlinear second order ordinary differential equations in is highly non trivial. However, this problem can be tackled using the information geometric diagonalization procedure introduced in [43]. The information metric tensor $\hat{g}\left(\mu_{1}, \ldots \mu_{l} ; \sigma_{1}, \ldots, \sigma_{l}\right) \stackrel{\text { def }}{=} \hat{g}(\Theta)$ in (8) is symmetric and therefore diagonalizable. The eigenvalues of such matrix are,

$$
\alpha_{ \pm}\left(r_{k}\right) \stackrel{\text { def }}{=} \frac{3 \pm \sqrt{\Delta\left(r_{k}\right)}}{2}, \Delta\left(r_{k}\right)=1+4 r_{k}^{2}, \text { with } k=1, \ldots, l .
$$

The eigenvectors $\Theta_{+}^{(k)} \stackrel{\text { def }}{=} \Theta_{+}\left(r_{k}\right)$ and $\Theta_{-}^{(k)} \stackrel{\text { def }}{=} \Theta_{-}(r)$ corresponding to $\alpha_{+}\left(r_{k}\right)$ and $\alpha_{-}\left(r_{k}\right)$, respectively, are,

$$
\Theta_{+}\left(r_{k}\right)=\left(\begin{array}{c}
1 \\
\frac{1+\sqrt{\Delta\left(r_{k}\right)}}{2 r_{k}}
\end{array}\right) \text { and, } \Theta_{-}\left(r_{k}\right)=\left(\begin{array}{c}
1 \\
\frac{1-\sqrt{\Delta\left(r_{k}\right)}}{2 r_{k}}
\end{array}\right) \text { with } k=1, \ldots, l .
$$


The diagonalized information matrix $\left[\hat{g}^{\prime}(\Theta(\tilde{\Theta}))\right]_{\mathcal{B}_{\text {new }}}$ in the new basis $\mathcal{B}_{\text {new }}$ satisfies the following relation,

$$
[\hat{g}(\Theta)]_{\mathcal{B}_{\text {old }}}=E_{2 l \times 2 l}\left(r_{1}, \ldots, r_{l}\right)\left[\hat{g}^{\prime}(\Theta(\tilde{\Theta}))\right]_{\mathcal{B}_{\text {new }}} E_{2 l \times 2 l}^{-1}\left(r_{1}, \ldots, r_{l}\right) \text {, }
$$

where, in an explicit way, we obtain

$$
\left[\hat{g}^{\prime}(\Theta(\tilde{\Theta}))\right]_{\mathcal{B}_{\text {new }}}=\left(\begin{array}{cccc}
D_{2 \times 2}^{(1)} & 0 & 0 & 0 \\
0 & \cdot & 0 & 0 \\
0 & 0 & \cdot & 0 \\
0 & 0 & 0 & D_{2 \times 2}^{(l)}
\end{array}\right)
$$

with the two-dimensional diagonal matrices $D_{2 \times 2}^{(k)}$ defined as,

$$
\left[D_{2 \times 2}^{(k)}\right] \stackrel{\text { def }}{=} \frac{1}{\sigma_{k}^{2}\left(\tilde{\mu}_{k}, \tilde{\sigma}_{k}\right)}\left(\begin{array}{cc}
\frac{3-\sqrt{\Delta\left(r_{k}\right)}}{2} & 0 \\
0 & \frac{3+\sqrt{\Delta\left(r_{k}\right)}}{2}
\end{array}\right) \text { with } k=1, \ldots, l .
$$

The columns of the matrix $E_{2 l \times 2 l}\left(r_{1}, \ldots, r_{l}\right)$ encode the eigenvectors of $[\hat{g}(\Theta)]_{\mathcal{B}_{\text {old }}}$, $\Theta_{+}^{(k)}$ and $\Theta_{-}^{(k)}$ and is given by,

$$
\left[E\left(r_{1}, \ldots, r_{l}\right)\right]_{2 l \times 2 l}=\left(\begin{array}{cccc}
E_{2 \times 2}^{(1)} & 0 & 0 & 0 \\
0 & \cdot & 0 & 0 \\
0 & 0 & \cdot & 0 \\
0 & 0 & 0 & E_{2 \times 2}^{(l)}
\end{array}\right)
$$

where the two-dimensional matrices $E_{2 \times 2}^{(k)}$ are,

$$
\left[E_{2 \times 2}^{(k)}\right] \stackrel{\text { def }}{=}\left(\begin{array}{cc}
1 & 1 \\
\frac{1-\sqrt{\Delta\left(r_{k}\right)}}{2 r_{k}} & \frac{1+\sqrt{\Delta\left(r_{k}\right)}}{2 r_{k}}
\end{array}\right)
$$

The relevance of $E_{2 l \times 2 l}\left(r_{1}, \ldots, r_{l}\right)$ (and its inverse) is in expressing the set of macrovariables $\left(\mu_{1}, \ldots \mu_{l} ; \sigma_{1}, \ldots, \sigma_{l}\right)$ in terms of the new statistical variables $\left(\tilde{\mu}_{1}, \ldots, \tilde{\mu}_{l} ; \tilde{\sigma}_{1}, \ldots, \tilde{\sigma}_{l}\right)$,

$$
g_{i j}\left(\mu_{1}, \ldots \mu_{l} ; \sigma_{1}, \ldots, \sigma_{l}\right) \stackrel{\operatorname{diag}}{\longrightarrow} g_{i j}^{\prime}\left(\tilde{\mu}_{1}, \ldots, \tilde{\mu}_{l} ; \tilde{\sigma}_{1}, \ldots, \tilde{\sigma}_{l}\right) \text {. }
$$

From differential geometry arguments [45], it follows that

$$
\left(\begin{array}{c}
\partial_{\tilde{\mu}_{1}} \\
\cdot \\
\cdot \\
\partial_{\tilde{\sigma}_{l}}
\end{array}\right)=E_{2 l \times 2 l}\left(r_{1}, \ldots, r_{l}\right)\left(\begin{array}{c}
\partial_{\mu_{1}} \\
\cdot \\
\cdot \\
\partial_{\sigma_{l}}
\end{array}\right) \text { and, }\left(\begin{array}{c}
\mu_{1} \\
\cdot \\
\cdot \\
\sigma_{l}
\end{array}\right)=E_{2 l \times 2 l}\left(r_{1}, \ldots, r_{k}\right)\left(\begin{array}{c}
\tilde{\mu}_{1} \\
\cdot \\
\cdot \\
\tilde{\sigma}_{l}
\end{array}\right) .
$$

Substituting (20) in (22), we finally obtain the formal relation between the old and new set of macrovariables labelling the $2 l$-dimensional macrostates $\Theta$ of the embedded Gaussian statistical model in presence of non-trivial off-diagonal terms,

$$
\mu_{k}\left(\tilde{\mu}_{k}, \tilde{\sigma}_{k}\right) \stackrel{\text { def }}{=} \tilde{\mu}_{k}+\tilde{\sigma}_{k} \text { and, } \sigma\left(\tilde{\mu}_{k}, \tilde{\sigma}_{k}\right) \stackrel{\text { def }}{=} \frac{1-\sqrt{\Delta\left(r_{k}\right)}}{2 r_{k}} \tilde{\mu}_{k}+\frac{1+\sqrt{\Delta\left(r_{k}\right)}}{2 r_{k}} \tilde{\sigma}_{k} \text {, with } k=1, \ldots, l .
$$

After having introduced the information geometric diagonalization procedure, the new line element $d s^{2}\left(\tilde{\mu}_{1}, \ldots, \tilde{\mu}_{l} ; \tilde{\sigma}_{1}, \ldots, \tilde{\sigma}_{l}\right)$ to be considered becomes,

$$
d s^{\prime 2}\left(\tilde{\mu}_{1}, \ldots, \tilde{\mu}_{l} ; \tilde{\sigma}_{1}, \ldots, \tilde{\sigma}_{l}\right)=\sum_{k=1}^{l}\left[\frac{\alpha_{-}\left(r_{k}\right)}{\left[a_{0}\left(r_{k}\right) \tilde{\mu}_{k}+a_{1}\left(r_{k}\right) \tilde{\sigma}_{k}\right]^{2}} d \tilde{\mu}_{k}^{2}+\frac{\alpha_{+}\left(r_{k}\right)}{\left[a_{0}\left(r_{k}\right) \tilde{\mu}_{k}+a_{1}\left(r_{k}\right) \tilde{\sigma}_{k}\right]^{2}} d \tilde{\sigma}_{k}^{2}\right],
$$

where,

$$
\alpha_{ \pm}\left(r_{k}\right) \stackrel{\text { def }}{=} \frac{3 \pm \sqrt{\Delta\left(r_{k}\right)}}{2}, a_{0}\left(r_{k}\right) \stackrel{\text { def }}{=} \frac{1-\sqrt{\Delta\left(r_{k}\right)}}{2 r_{k}}, a_{1}\left(r_{k}\right) \stackrel{\text { def }}{=} \frac{1+\sqrt{\Delta\left(r_{k}\right)}}{2 r_{k}} \text { and, } \Delta\left(r_{k}\right) \stackrel{\text { def }}{=} 1+4 r_{k}^{2} \text {. }
$$


Notice that $d s^{\prime 2}\left(\tilde{\mu}_{1}, \ldots, \tilde{\mu}_{l} ; \tilde{\sigma}_{1}, \ldots, \tilde{\sigma}_{l}\right)$ can be rewritten as,

$$
d s^{2}\left(\tilde{\mu}_{1}, ., \tilde{\mu}_{l} ; \tilde{\sigma}_{1}, ., \tilde{\sigma}_{l}\right)=\sum_{k=1}^{l}\left[\frac{\alpha_{-}\left(r_{k}\right)}{\left[a_{1}\left(r_{k}\right)\right]^{2}} \frac{1}{\tilde{\sigma}_{k}^{2}} \frac{1}{\left(1+\frac{a_{0}\left(r_{k}\right)}{a_{1}\left(r_{k}\right)} \frac{\tilde{\mu}_{k}}{\tilde{\sigma}_{k}}\right)^{2}} d \tilde{\mu}_{k}^{2}+\frac{\alpha_{+}\left(r_{k}\right)}{\left[a_{1}\left(r_{k}\right)\right]^{2}} \frac{1}{\tilde{\sigma}_{k}^{2}} \frac{1}{\left(1+\frac{a_{0}\left(r_{k}\right)}{a_{1}\left(r_{k}\right)} \frac{\tilde{\mu}_{k}}{\tilde{\sigma}_{k}}\right)^{2}} d \tilde{\sigma}_{k}^{2}\right] .
$$

As a working hypothesis, we assume that $\frac{a_{0}\left(r_{k}\right)}{a_{1}\left(r_{k}\right)} \frac{\tilde{\mu}_{k}(\tau)}{\tilde{\sigma}_{k}(\tau)} \ll 1$ for $\tau \gg 1$ and for each $k=1, . ., l$. Stated otherwise, we assume that

$$
\lim _{\tau \rightarrow \infty}\left[\frac{\tilde{\mu}_{k}(\tau)}{\tilde{\sigma}_{k}(\tau)}\right] \ll \min _{r \in(0,1)}\left|\frac{a_{1}\left(r_{k}\right)}{a_{0}\left(r_{k}\right)}\right|=\min _{r_{k} \in(0,1)}\left|\frac{1+\sqrt{1+4 r_{k}^{2}}}{1-\sqrt{1+4 r_{k}^{2}}}\right| \simeq 2.6 .
$$

Then, in the asymptotic long-time limit [46], the notion of distinguishability between probability distributions on the diagonalized statistical manifold is quantified by the following line element,

$$
d s^{\prime 2}\left(\tilde{\mu}_{1}, ., \tilde{\mu}_{l} ; \tilde{\sigma}_{1}, . ., \tilde{\sigma}_{l}\right)=\frac{\alpha_{-}\left(r_{k}\right)}{\left[a_{1}\left(r_{k}\right)\right]^{2}} \frac{1}{\tilde{\sigma}_{k}^{2}} d \tilde{\mu}_{k}^{2}+\frac{\alpha_{+}\left(r_{k}\right)}{\left[a_{1}\left(r_{k}\right)\right]^{2}} \frac{1}{\tilde{\sigma}_{k}^{2}} d \tilde{\sigma}_{k}^{2} .
$$

Recall that the Christoffel connection coefficients $\Gamma_{i j}^{n}$ are defined as,

$$
\Gamma_{i j}^{n} \stackrel{\text { def }}{=} \frac{1}{2} g^{n m}\left(\partial_{i} g_{m j}+\partial_{j} g_{i m}-\partial_{m} g_{i j}\right) \text {. }
$$

Substituting the metric tensor components from (28) into (29), it turns out that the only non-zero connection coefficients are given by,

$$
\left(\Gamma_{12}^{1}\right)^{k}=-\frac{1}{\sigma_{k}},\left(\Gamma_{11}^{2}\right)^{k}=\frac{\alpha_{-}\left(r_{k}\right)}{\alpha_{+}\left(r_{k}\right)} \frac{1}{\sigma_{k}},\left(\Gamma_{22}^{2}\right)^{k}=-\frac{1}{\sigma_{k}},
$$

where $k=1, . ., l$. Therefore the set of coupled nonlinear ordinary differential equations satisfied by the geodesic trajectories becomes,

$$
\frac{d^{2} \tilde{\mu}_{k}}{d \tau^{2}}-\frac{2}{\tilde{\sigma}} \frac{d \tilde{\mu}_{k}}{d \tau} \frac{d \tilde{\sigma}_{k}}{d \tau}=0, \frac{d^{2} \tilde{\sigma}_{k}}{d \tau^{2}}+\frac{\alpha_{-}\left(r_{k}\right)}{\alpha_{+}\left(r_{k}\right)} \frac{1}{\tilde{\sigma}}\left(\frac{d \tilde{\mu}_{k}}{d \tau}\right)^{2}-\frac{1}{\tilde{\sigma}_{k}}\left(\frac{d \tilde{\sigma}_{k}}{d \tau}\right)^{2}=0
$$

Notice that in the limit of $r_{k} \rightarrow 0, \frac{\alpha_{-}\left(r_{k}\right)}{\alpha_{+}\left(r_{k}\right)} \rightarrow \frac{1}{2}$ and the system of equations (31) describing the asymptotic behavior of maximally probable trajectories on the diagonalized manifold becomes the standard two-dimensional Gaussian system of nonlinear coupled ordinary differential equations studied in [36]. In order to further simplify the integration of (31), consider the following (invertible) change of variables,

$$
\left(\tilde{\mu}_{k}, \tilde{\sigma}_{k}\right) \longrightarrow\left(\mu_{k}^{\prime}\left(\tilde{\mu}_{k}, \tilde{\sigma}_{k}\right)=\sqrt{\frac{2 \alpha_{-}\left(r_{k}\right)}{\alpha_{+}\left(r_{k}\right)}} \tilde{\mu}_{k}, \sigma_{k}^{\prime}\left(\tilde{\mu}_{k}, \tilde{\sigma}_{k}\right)=\tilde{\sigma}_{k}\right) .
$$

Substituting (32) into (31), the coupled system of nonlinear differential equations to be integrated becomes,

$$
\frac{d^{2} \mu_{k}^{\prime}}{d \tau^{2}}-\frac{2}{\sigma^{\prime}} \frac{d \mu_{k}^{\prime}}{d \tau} \frac{d \sigma_{k}^{\prime}}{d \tau}=0, \frac{d^{2} \sigma_{k}^{\prime}}{d \tau^{2}}+\frac{1}{2 \sigma_{k}^{\prime}}\left(\frac{d \mu_{k}^{\prime}}{d \tau}\right)^{2}-\frac{1}{\sigma_{k}^{\prime}}\left(\frac{d \sigma_{k}^{\prime}}{d \tau}\right)^{2}=0 .
$$

Integrating (33) leads to the following geodesic trajectories,

$$
\mu_{k}^{\prime}(\tau)=\frac{\Xi_{k}^{2}}{2 \lambda_{k}} \frac{1}{\exp \left(-2 \lambda_{k} \tau\right)+\frac{\Xi_{k}^{2}}{8 \lambda_{k}^{2}}}-4 \lambda_{k}, \sigma_{k}^{\prime}(\tau)=\frac{\Xi_{k} \exp \left(-\lambda_{k} \tau\right)}{\exp \left(-2 \lambda_{k} \tau\right)+\frac{\Xi_{k}^{2}}{8 \lambda_{k}^{2}}}
$$

where $\Xi_{k}$ and $\lambda_{k}$ are real and positive constants of integration [36]. Using (23) and (32), we have

$$
\mu_{k}\left(\mu_{k}^{\prime}, \sigma_{k}^{\prime}\right) \stackrel{\text { def }}{=} \sqrt{\frac{\alpha_{+}\left(r_{k}\right)}{2 \alpha_{-}\left(r_{k}\right)}} \mu_{k}^{\prime}+\sigma_{k}^{\prime} \text { and, } \sigma_{k}\left(\mu_{k}^{\prime}, \sigma_{k}^{\prime}\right) \stackrel{\text { def }}{=} \frac{1-\sqrt{\Delta\left(r_{k}\right)}}{2 r_{k}} \sqrt{\frac{\alpha_{+}\left(r_{k}\right)}{2 \alpha_{-}\left(r_{k}\right)}} \mu_{k}^{\prime}+\frac{1+\sqrt{\Delta\left(r_{k}\right)}}{2 r_{k}} \sigma_{k}^{\prime} .
$$


Notice that our working hypothesis (27) is satisfied since we have,

$$
\lim _{\tau \rightarrow \infty} \frac{\tilde{\mu}_{k}(\tau)}{\tilde{\sigma}_{k}(\tau)}=\left(\sqrt{\frac{\alpha_{+}\left(r_{k}\right)}{2 \alpha_{-}\left(r_{k}\right)}}\right) \cdot \lim _{\tau \rightarrow \infty} \frac{\mu_{k}^{\prime}(\tau)}{\sigma_{k}^{\prime}(\tau)} \propto \exp \left(-\lambda_{k} \tau\right) \stackrel{\tau \rightarrow \infty}{\longrightarrow} 0 .
$$

Finally, in terms of the original macrovariables $\left(\mu_{k}, \sigma_{k}\right)$, the geodesic trajectories become,

$$
\begin{gathered}
\mu_{k}\left(\tau ; r_{k}\right)=\sqrt{\frac{\alpha_{+}\left(r_{k}\right)}{2 \alpha_{-}\left(r_{k}\right)}}\left[\frac{\Xi_{k}^{2}}{2 \lambda_{k}} \frac{1}{\exp \left(-2 \lambda_{k} \tau\right)+\frac{\Xi_{k}^{2}}{8 \lambda_{k}^{2}}}-4 \lambda_{k}\right]+\frac{\Xi_{k} \exp \left(-\lambda_{k} \tau\right)}{\exp \left(-2 \lambda_{k} \tau\right)+\frac{\Xi_{k}^{2}}{8 \lambda_{k}^{2}}}, \\
\sigma_{k}\left(\tau ; r_{k}\right)=\frac{1-\sqrt{\Delta\left(r_{k}\right)}}{2 r_{k}} \sqrt{\frac{\alpha_{+}\left(r_{k}\right)}{2 \alpha_{-}\left(r_{k}\right)}}\left[\frac{\Xi_{k}^{2}}{2 \lambda_{k}} \frac{1}{\exp \left(-2 \lambda_{k} \tau\right)+\frac{\Xi_{k}^{2}}{8 \lambda_{k}^{2}}}-4 \lambda_{k}\right]+\frac{1+\sqrt{\Delta\left(r_{k}\right)}}{2 r_{k}} \frac{\Xi_{k} \exp \left(-\lambda_{k} \tau\right)}{\exp \left(-2 \lambda_{k} \tau\right)+\frac{\Xi_{k}^{2}}{8 \lambda_{k}^{2}}} .
\end{gathered}
$$

In our probabilistic macroscopic approach to dynamics, the geodesic trajectories in (37) represent the maximum probability paths on the $2 l$-dimensional embedded Gaussian statistical model.

\section{INFORMATION GEOMETRIC COMPLEXITY}

In our information geometric approach a relevant quantity that can be useful to study the degree of complexity characterizing information-constrained dynamical models is the information geometrodynamical entropy $\mathcal{S}_{\mathcal{M}_{s}}(\tau)$ (IGE) [36]. In what follows, we will briefly highlight the key-points leading to the construction of such quantity.

The elements (or points) $\{P(X \mid \Theta)\}$ of a $2 l$-dimensional curved statistical manifold $\mathcal{M}_{s}$ are parametrized using $2 l$-real valued variables $\left(\theta^{1}, \ldots, \theta^{2 l}\right)$,

$$
\mathcal{M}_{s} \stackrel{\text { def }}{=}\left\{P(X \mid \Theta): \Theta=\left(\theta^{1}, \ldots, \theta^{2 l}\right) \in \mathcal{D}_{\Theta}^{(\text {tot })}\right\} .
$$

The set $\mathcal{D}_{\Theta}^{(\text {tot })}$ is the entire parameter space (at disposal) and it is a subset of $\mathbb{R}^{2 l}$,

$$
\mathcal{D}_{\Theta}^{\text {(tot) }} \stackrel{\text { def }}{=} \bigotimes_{k=1}^{2 l} \mathcal{I}_{\theta^{k}}=\left(\mathcal{I}_{\theta^{1}} \otimes \mathcal{I}_{\theta^{2}} \ldots \otimes \mathcal{I}_{\theta^{2 l}}\right) \subseteq \mathbb{R}^{2 l}
$$

where $\mathcal{I}_{\theta^{k}}$ is a subset of $\mathbb{R}$ and represents the entire range of allowable values for the macrovariable $\theta^{k}$. For instance, considering the statistical manifold of one-dimensional Gaussian probability distributions parametrized as usual in terms of $\Theta=(\mu, \sigma)$, we obtain

$$
\mathcal{D}_{\Theta}^{(\text {tot })}=\mathcal{I}_{\mu} \otimes \mathcal{I}_{\sigma}=[(-\infty,+\infty) \otimes(0,+\infty)] \subseteq \mathbb{R}^{2} .
$$

In the IGAC, we are interested in a probabilistic description of the evolution of a given system in terms of its correspondent probability distribution on $\mathcal{M}_{s}$ which is homeomorphic to $\mathcal{D}_{\Theta}^{(\text {tot })}$. Assume we are interested in the evolution from $\tau_{\text {initial }}$ to $\tau_{\text {final }}$. Within the probabilistic description, this turns out to be equivalent to study the shortest path (or, in terms of the ME method [27 30], the maximally probable path) leading to $\Theta\left(\tau_{\text {final }}\right)$ from $\Theta\left(\tau_{\text {initial }}\right)$.

Is there a way to quantify the "complexity" of such path?. We have proposed that the IGE $\mathcal{S}_{\mathcal{M}_{s}}(\tau)$ is a good complexity quantifier [25, 26]. A suitable indicator of temporal complexity within the IGAC framework is provided by the information geometric entropy (IGE) $\mathcal{S}_{\mathcal{M}_{s}}(\tau)$ [36],

$$
\mathcal{S}_{\mathcal{M}_{s}}(\tau) \stackrel{\text { def }}{=} \log \widetilde{\operatorname{vol}}\left[\mathcal{D}_{\Theta}^{\text {(geodesic) }}(\tau)\right]
$$

The average dynamical statistical volume $\widetilde{\operatorname{vol}}\left[\mathcal{D}_{\Theta}^{\text {(geodesic) }}(\tau)\right]$ is defined as,

$$
\widetilde{\operatorname{vol}}\left[\mathcal{D}_{\Theta}^{\text {(geodesic) }}(\tau)\right] \stackrel{\text { def }}{=} \lim _{\tau \rightarrow \infty}\left(\frac{1}{\tau} \int_{0}^{\tau} d \tau^{\prime} \operatorname{vol}\left[\mathcal{D}_{\Theta}^{\text {(geodesic) }}\left(\tau^{\prime}\right)\right]\right)
$$


where the "tilde" symbol denotes the operation of temporal average. The volume $\operatorname{vol}\left[\mathcal{D}_{\Theta}^{(\text {geodesic) }}\left(\tau^{\prime}\right)\right]$ is given by,

$$
\operatorname{vol}\left[\mathcal{D}_{\Theta}^{\text {(geodesic) }}\left(\tau^{\prime}\right)\right] \stackrel{\text { def }}{=} \int_{\mathcal{D}_{\Theta}^{\text {(geodesic) }}\left(\tau^{\prime}\right)} \rho_{\left(\mathcal{M}_{s}, g\right)}\left(\theta^{1}, \ldots, \theta^{n}\right) d^{n} \Theta
$$

where $\rho_{\left(\mathcal{M}_{s}, g\right)}\left(\theta^{1}, \ldots, \theta^{n}\right)$ is the so-called Fisher density and is equal to the square root of the determinant $g=$ $\left|\operatorname{det}\left(g_{\mu \nu}\right)\right|$ of the metric tensor $g_{\mu \nu}(\Theta)$,

$$
\rho_{\left(\mathcal{M}_{s}, g\right)}\left(\theta^{1}, \ldots, \theta^{n}\right) \stackrel{\text { def }}{=} \sqrt{\left|g\left(\left(\theta^{1}, \ldots, \theta^{n}\right)\right)\right|} .
$$

The integration space $\mathcal{D}_{\Theta}^{\text {(geodesic) }}\left(\tau^{\prime}\right)$ in (43) is defined as follows,

$$
\mathcal{D}_{\Theta}^{\text {(geodesic) }}\left(\tau^{\prime}\right) \stackrel{\text { def }}{=}\left\{\Theta \equiv\left(\theta^{1}, \ldots, \theta^{n}\right): \theta^{k}(0) \leq \theta^{k} \leq \theta^{k}\left(\tau^{\prime}\right)\right\},
$$

where $k=1, . ., n$ and $\theta^{k} \equiv \theta^{k}(s)$ with $0 \leq s \leq \tau^{\prime}$ such that,

$$
\frac{d^{2} \theta^{k}(s)}{d s^{2}}+\Gamma_{l m}^{k} \frac{d \theta^{l}}{d s} \frac{d \theta^{m}}{d s}=0 .
$$

The integration space $\mathcal{D}_{\Theta}^{\text {(geodesic) }}\left(\tau^{\prime}\right)$ in (45) is a $2 l$-dimensional subspace of the whole (permitted) parameter space $\mathcal{D}_{\Theta}^{\text {(tot) }}$. The elements of $\mathcal{D}_{\Theta}^{\text {(geodesic) }}\left(\tau^{\prime}\right)$ are the $2 l$-dimensional macrovariables $\{\Theta\}$ whose components $\theta^{k}$ are bounded by specified limits of integration $\theta^{k}(0)$ and $\theta^{k}\left(\tau^{\prime}\right)$ with $k=1, . ., 2 l$. The limits of integration are obtained via integration of the $2 l$-dimensional set of coupled nonlinear second order ordinary differential equations characterizing the geodesic equations. Formally, the IGE $\mathcal{S}_{\mathcal{M}_{s}}(\tau)$ is defined in terms of an averaged parametric ( $\tau$ is the parameter) $2 l+1$-fold integral over the multidimensional geodesic paths connecting $\Theta(0)$ to $\Theta(\tau)$. In our information geometric approach, the information geometric complexity $\widetilde{\operatorname{vol}}\left[\mathcal{D}_{\Theta}^{\text {(geodesic) }}(\tau)\right]$ represents a statistical measure of complexity of the macroscopic path $\Theta \stackrel{\text { def }}{=} \Theta(\tau)$ on $\mathcal{M}_{S}$ connecting the initial and final macrostates $\Theta_{i}$ and $\Theta_{f}$, respectively. The path $\Theta(\tau)$ is obtained via integration of the geodesic equation on $\mathcal{M}_{S}$ generated by the universal ME updating method. At a discrete level, the path $\Theta(\tau)$ can be described in terms of an infinite continuos sequence of intermediate macroscopic states, $\Theta(\tau)=\left[\Theta_{i}, \ldots, \Theta_{\bar{k}-1}, \Theta_{\bar{k}}, \Theta_{\bar{k}+1}, \ldots, \Theta_{f}\right]$ with $\Theta_{j}=\Theta\left(\tau_{j}\right)$, determined via the logarithmic relative entropy maximization procedure subjected to well-specified normalization and information constraints. The nature of such constraints defines the (correlational) structure of the underlying probability distribution on the particular curved statistical manifold $\mathcal{M}_{S}$. In other words, the correlational structure that may emerge into our information-geometric statistical models has its origin in the valuable information about the microscopic degrees of freedom of the actual physical systems. It emerges in the ME maximization procedure via integration of the geodesic equations defined on $\mathcal{M}_{S}$ and it is finally quantified in terms of the intuitive notion of volume growth via the information geometric complexity $\widetilde{\text { vol }}\left[\mathcal{D}_{\Theta}^{\text {(geodesic) }}(\tau)\right]$ or, in entropic terms by the IGE $\mathcal{S}_{\mathcal{M}_{S}}(\tau)$. The information geometric complexity is then interpreted as the volume of the statistical macrospace explored in the asymptotic limit by the system in its complex evolution from $\Theta_{i}$ to $\Theta_{f}$. Otherwise, upon a suitable normalization procedure that makes the information geometric complexity an adimensional quantity, it represents the number of accessible macrostates (with coordinates living in the accessed parameter space $\mathcal{D}_{\Theta}^{\text {(geodesic) }}(\tau)$ ) explored by the system in its evolution from $\Theta_{i}$ to $\Theta_{f}$.

For the model defined in (8), $\mathcal{V}_{\mathcal{M}_{s}}(\tau)$ becomes,

$$
\mathcal{V}_{\mathcal{M}_{s}}(\tau) \equiv \widetilde{\operatorname{vol}}\left[\mathcal{D}_{\Theta}^{\text {(geodesic) }}(\tau)\right] \stackrel{\text { def }}{=} \frac{1}{\tau} \int_{0}^{\tau} d \tau^{\prime}\left(\int_{\Theta_{i}(0)}^{\Theta_{f}\left(\tau^{\prime}\right)} \sqrt{g} d^{2 l} \Theta\right)
$$

where $g \stackrel{\text { def }}{=} \operatorname{det}\left[g_{i j}(\Theta)\right]_{2 l \times 2 l}$ is the determinant of the block-diagonal matrix in (9),

$$
g\left(r_{1}, . ., r_{l}\right)=\prod_{k=1}^{l} \operatorname{det}\left(M_{2 \times 2}^{(k)}\right)=\prod_{k=1}^{l}\left[\frac{2-r_{k}^{2}}{\sigma_{k}^{2}}\right] .
$$


The geodesic paths $\Theta(\tau)=\left(\mu_{1}\left(\tau ; r_{1}\right), \sigma_{1}\left(\tau ; r_{1}\right) ; . . ; \mu_{l}\left(\tau ; r_{l}\right), \sigma_{l}\left(\tau ; r_{l}\right)\right)$ are given in (37). Substituting them into (47), we get,

$$
\mathcal{V}_{\mathcal{M}_{s}}\left(\tau ; \lambda_{1}, . ., \lambda_{k}\right)=\prod_{k=1}^{l}\left\{\frac{\sqrt{2-r_{k}^{2}}}{\tau} \int^{\tau}\left[\frac{\Xi_{k} \exp \left(-\lambda_{k} \tau^{\prime}\right)-4 \lambda_{k} \sqrt{\frac{\alpha_{+}\left(r_{k}\right)}{2 \alpha_{-}\left(r_{k}\right)}} \exp \left(-2 \lambda_{k} \tau^{\prime}\right)}{\frac{1+\sqrt{\Delta\left(r_{k}\right)}}{2 r_{k}} \Xi_{k} \exp \left(-\lambda_{k} \tau^{\prime}\right)-4 \lambda_{k} \sqrt{\frac{\alpha_{+}\left(r_{k}\right)}{2 \alpha_{-}\left(r_{k}\right)}} \frac{1-\sqrt{\Delta\left(r_{k}\right)}}{2 r_{k}} \exp \left(-2 \lambda_{k} \tau^{\prime}\right)}\right] d \tau^{\prime}\right\}
$$

For the sake of simplicity, let us introduce the following substitutions,

$$
A_{k} \stackrel{\text { def }}{=} \Xi_{k}, B_{k} \stackrel{\text { def }}{=}-4 \lambda_{k} \sqrt{\frac{\alpha_{+}\left(r_{k}\right)}{2 \alpha_{-}\left(r_{k}\right)}}, C_{k} \stackrel{\text { def }}{=} \frac{1+\sqrt{\Delta\left(r_{k}\right)}}{2 r_{k}} \Xi_{k}, D_{k} \stackrel{\text { def }}{=}-4 \lambda_{k} \sqrt{\frac{\alpha_{+}\left(r_{k}\right)}{2 \alpha_{-}\left(r_{k}\right)}} \frac{1-\sqrt{\Delta\left(r_{k}\right)}}{2 r_{k}} .
$$

Then, the integral defining $\mathcal{V}_{\mathcal{M}_{s}}\left(\tau ; \lambda_{1}, . ., \lambda_{k}\right)$ becomes,

$$
\mathcal{V}_{\mathcal{M}_{s}}\left(\tau ; \lambda_{1}, . ., \lambda_{k}\right)=\prod_{k=1}^{l}\left\{\frac{\sqrt{2-r_{k}^{2}}}{\tau} \int^{\tau}\left[\frac{A_{k} e^{-\lambda_{k} \tau^{\prime}}+B_{k} e^{-2 \lambda_{k} \tau^{\prime}}}{C_{k} e^{-\lambda_{k} \tau^{\prime}}+D_{k} e^{-2 \lambda_{k} \tau^{\prime}}} d \tau^{\prime}\right] d \tau^{\prime}\right\}
$$

Upon integration, we get

$$
\int^{\tau} \frac{A_{k} e^{-\lambda_{k} \tau^{\prime}}+B_{k} e^{-2 \lambda_{k} \tau^{\prime}}}{C_{k} e^{-\lambda_{k} \tau^{\prime}}+D_{k} e^{-2 \lambda_{k} \tau^{\prime}}} d \tau^{\prime}=\frac{1}{\lambda_{k}}\left(\frac{A_{k}}{C_{k}}-\frac{B_{k}}{D_{k}}\right) \ln \left[\frac{D_{k}+C_{k} e^{\lambda_{k} \tau}}{D_{k} e^{\lambda_{k} \tau}}\right]+\frac{A_{k}}{C_{k}} \tau \stackrel{\tau \rightarrow \infty}{\approx} \frac{1}{\lambda_{k}}\left(\frac{A_{k}}{C_{k}}-\frac{B_{k}}{D_{k}}\right) \ln \frac{C_{k}}{D_{k}}+\frac{A_{k}}{C_{k}} \tau
$$

and substituting (52) into (51), we obtain

$$
\mathcal{V}_{\mathcal{M}_{s}}\left(\tau ; \lambda_{1}, . ., \lambda_{k}\right)=\prod_{k=1}^{l}\left\{\sqrt{2-r_{k}^{2}}\left[\frac{A_{k}}{C_{k}}+\frac{1}{\lambda_{k}}\left(\frac{A_{k}}{C_{k}}-\frac{B_{k}}{D_{k}}\right) \ln \frac{C_{k}}{D_{k}} \frac{1}{\tau}\right]\right\} .
$$

Introducing again the original parameters in (50), we finally get

$$
\mathcal{V}_{\mathcal{M}_{s}}\left(\tau ; \lambda_{1}, . ., \lambda_{k}\right)=\prod_{k=1}^{l}\left\{\frac{2 r_{k} \sqrt{2-r_{k}^{2}}}{1+\sqrt{\Delta\left(r_{k}\right)}}+\left[\frac{\left(2 r_{k} \sqrt{2-r_{k}^{2}}\right)}{\left(1+\sqrt{\Delta\left(r_{k}\right)}\right) \lambda_{k}}-\frac{2 r_{k} \sqrt{2-r_{k}^{2}}}{\left(1-\sqrt{\Delta\left(r_{k}\right)}\right) \lambda_{k}}\right] \frac{\ln \Sigma\left(r_{k}, \lambda_{k}, \alpha_{ \pm}\right)}{\tau}\right\}
$$

where the strictly positive function $\Sigma\left(r_{k}, \lambda_{k}, \alpha_{ \pm}\right)$is given by,

$$
\Sigma\left(r_{k}, \lambda_{k}, \alpha_{ \pm}\right) \stackrel{\text { def }}{=} \frac{\frac{1+\sqrt{\Delta\left(r_{k}\right)}}{2 r_{k}}}{-4 \lambda_{k} \sqrt{\frac{\alpha_{+}\left(r_{k}\right)}{2 \alpha_{-}\left(r_{k}\right)}} \frac{1-\sqrt{\Delta\left(r_{k}\right)}}{2 r_{k}}}>0, \forall r_{k} \in(0,1) .
$$

Finally, inserting (54) into (41), the IGE $\mathcal{S}_{\mathcal{M}_{s}}(\tau)$ becomes,

$$
\mathcal{S}_{\mathcal{M}_{s}}\left(\tau ; \lambda_{1}, . ., \lambda_{k}\right)=\sum_{k=1}^{l} \log \left\{\frac{2 r_{k} \sqrt{2-r_{k}^{2}}}{1+\sqrt{\Delta\left(r_{k}\right)}}+\left[\frac{2 r_{k} \sqrt{2-r_{k}^{2}}}{\left(1+\sqrt{\Delta\left(r_{k}\right)}\right) \lambda_{k}}-\frac{2 r_{k} \sqrt{2-r_{k}^{2}}}{\left(1-\sqrt{\Delta\left(r_{k}\right)}\right) \lambda_{k}}\right] \frac{\ln \Sigma\left(r_{k}, \lambda_{k}, \alpha_{ \pm}\right)}{\tau}\right\} .
$$

With a suitable change of notation, equation (56) can be rewritten in a more elegant way as follows,

$$
\mathcal{S}_{\mathcal{M}_{s}}\left(\tau ; \lambda_{1}, . ., \lambda_{k}\right) \stackrel{\tau \rightarrow \infty}{\sim} \sum_{k=1}^{l} \log \left[\Lambda_{1}\left(r_{k}\right)+\frac{\Lambda_{2}\left(r_{k}, \lambda_{k}\right)}{\tau}\right]
$$

where,

$$
\Lambda_{1}\left(r_{k}\right) \stackrel{\text { def }}{=} \frac{2 r_{k} \sqrt{2-r_{k}^{2}}}{1+\sqrt{1+4 r_{k}^{2}}}, \Lambda_{2}\left(r_{k}, \lambda\right) \stackrel{\text { def }}{=} \frac{\sqrt{\left(1+4 r_{k}^{2}\right)\left(2-r_{k}^{2}\right)}}{r_{k}} \frac{\ln \Sigma\left(r_{k}, \lambda_{k}, \alpha_{ \pm}\right)}{\lambda_{k}}, \alpha_{ \pm}\left(r_{k}\right) \stackrel{\text { def }}{=} \frac{3 \pm \sqrt{1+4 r_{k}^{2}}}{2} .
$$

As stated above, $\Sigma\left(r_{k}, \lambda_{k}, \alpha_{ \pm}\right)$is a strictly positive function of its arguments. It appears that the introduction of embedding constraints between the macrovariables of the larger Gaussian statistical model leads to the emergence of an 
asymptotic information geometric compression of the explored statistical macrostates on the embedded configuration manifold $\mathcal{M}_{s}$ in its evolution between the initial and final macrostates. This result, thought for a special (not general) case, leads to interesting conclusions. The presence of constraints between macroscopic pieces of relevant information on the microscopic degrees of freedom of a complex system allows for an information geometric probabilistic description whose complexity, measured in terms of $\mathcal{S}_{\mathcal{M}_{s}}\left(\right.$ or $\mathcal{V}_{\mathcal{M}_{s}}$ ), decays in a power law way. Asymptotically, the complexity reaches a saturation value characterized solely by the strength of such macroscopic correlational coefficients. The relevance of such results becomes even more clear if compared to what happens for the $4 l$-dimensional (uncorrelated and larger) Gaussian statistical model [36]. We will mention this comparison in our final remarks. For $r_{k}=r_{s}, \forall k$, $s=1, \ldots, l$, the information geometric entropy $\mathcal{S}_{\mathcal{M}_{s}}(\tau ; l, \lambda, r)$ becomes,

$$
\mathcal{S}_{\mathcal{M}_{s}}(\tau ; l, \lambda, r) \stackrel{\tau \rightarrow \infty}{\sim} \log \left[\Lambda_{1}(r)+\frac{\Lambda_{2}(r, \lambda)}{\tau}\right]^{l}
$$

Therefore, the information geometric complexity presents a power law decay where the power is related to cardinality $l$ of the microscopic degrees of freedom characterized by correlated pieces of macroscopic information and it reaches a saturation value quantified by the set of coefficients $\left\{r_{k}\right\}$.

\section{JACOBI-LEVI-CIVITA EQUATION AND JACOBI FIELDS}

The Jacobi-Levi-Civita (JLC) equation of geodesic deviation is a complicated second-order system of linear ordinary differential equations. It describes the geodesic spread on curved manifolds of a pair of nearby freely falling particles travelling on trajectories $\Theta^{\rho}(\tau)$ and $\Theta^{\prime \rho}(\tau) \stackrel{\text { def }}{=} \Theta^{\rho}(\tau)+\delta \Theta^{\rho}(\tau)$. The JLC equation is given by [47],

$$
\frac{\mathcal{D}^{2} J^{k}}{\mathcal{D} \tau^{2}}+\mathcal{R}_{n m l}^{k} \frac{\partial \Theta^{n}}{\partial \tau} J^{m} \frac{\partial \Theta^{l}}{\partial \tau}=0
$$

with $k=1, . ., 2 l$ and where the covariant derivatives $\frac{\mathcal{D} \Theta^{\mu}(\tau)}{\mathcal{D} \tau}$ along the curve $\Theta^{\mu}(\tau)$ are defined as,

$$
\frac{\mathcal{D} \Theta^{\mu}(\tau)}{\mathcal{D} \tau} \stackrel{\text { def }}{=} \frac{d \Theta^{\mu}(\tau)}{d \tau}+\Gamma_{\nu \rho}^{\mu} \frac{d \Theta^{\rho}}{d \tau} \Theta^{\nu}
$$

The Jacobi vector field components $J^{k}$ are given by,

$$
J^{k} \equiv \delta_{\lambda_{k}} \Theta^{k} \stackrel{\text { def }}{=}\left(\frac{\partial \Theta^{k}\left(\tau ; \lambda_{k}\right)}{\partial \lambda_{k}}\right)_{\tau} \delta \lambda_{k}
$$

and $\mathcal{R}_{\alpha \beta \gamma \delta}$ is the Riemann curvature tensor defined as [47],

$$
\mathcal{R}_{\mu \nu \rho}^{\alpha} \stackrel{\text { def }}{=} \partial_{\nu} \Gamma_{\mu \rho}^{\alpha}-\partial_{\rho} \Gamma_{\mu \nu}^{\alpha}+\Gamma_{\beta \nu}^{\alpha} \Gamma_{\mu \rho}^{\beta}-\Gamma_{\beta \rho}^{\alpha} \Gamma_{\mu \nu}^{\beta} .
$$

In the $2 l$-dimensional case $J=\left\{J^{k}\right\}_{k=1, . ., 2 l}$ represents how geodesics, in a 1-parameter family of geodesics, are separating. The covariant derivative $\frac{\mathcal{D}^{2} J^{\mu}}{\mathcal{D} \tau^{2}}$ in (60) is defined as [48],

$$
\frac{\mathcal{D}^{2} J^{\mu}}{\mathcal{D} \tau^{2}}=\frac{d^{2} J^{\mu}}{d \tau^{2}}+2 \Gamma_{\alpha \beta}^{\mu} \frac{d J^{\alpha}}{d \tau} \frac{d \Theta^{\beta}}{d \tau}+\Gamma_{\alpha \beta}^{\mu} J^{\alpha} \frac{d^{2} \Theta^{\beta}}{d \tau^{2}}+\Gamma_{\alpha \beta, \nu}^{\mu} \frac{d \Theta^{\nu}}{d \tau} \frac{d \Theta^{\beta}}{d \tau} J^{\alpha}+\Gamma_{\alpha \beta}^{\mu} \Gamma_{\rho \sigma}^{\alpha} \frac{d \Theta^{\sigma}}{d \tau} \frac{d \Theta^{\beta}}{d \tau} J^{\rho} .
$$

Equation (60) forms a system of $2 l$ coupled ordinary differential equations linear in the components of the deviation vector field (62) but nonlinear in derivatives of the metric tensor $g_{i j}(\Theta)$. It describes the linearized geodesic flow: the linearization ignores the relative velocity of the geodesics. When the geodesics are neighboring but their relative velocity is arbitrary, the corresponding geodesic deviation equation is the so-called generalized Jacobi equation [49]. The nonlinearity is due to the existence of velocity-dependent terms in the system. Neighboring geodesics accelerate relative to each other with a rate directly measured by the curvature tensor $\mathcal{R}_{\alpha \beta \gamma \delta}$.

Multiplying both sides of (60) by $g_{i j}(\Theta)$ and using the standard symmetry properties of the Riemann curvature tensor, the geodesic deviation equation becomes,

$$
g_{j i} \frac{\mathcal{D}^{2} J^{i}}{\mathcal{D} \tau^{2}}+\mathcal{R}_{l m k j} \frac{\partial \Theta^{k}}{\partial \tau} J^{m} \frac{\partial \Theta^{l}}{\partial \tau}=0 .
$$


After some algebra, it follows that the only non-zero Riemann tensor components are given by,

$$
\left(\mathcal{R}_{1212}\right)^{k}=-\frac{\alpha_{-}\left(r_{k}\right)}{a_{1}^{2}\left(r_{k}\right)} \frac{1}{\sigma_{k}^{2}}, k=1, . ., 2 l .
$$

In the model considered, the Jacobi field $\tilde{J}_{\mathcal{M}_{s}}$ has $2 l$-components $\left\{\tilde{J}^{\mu}\right\}_{\mu=1, . ., 2 l}$ that can be grouped into $l$-pairs as follows,

$$
\tilde{J}_{\mathcal{M}_{s}} \leftrightarrow\left(\tilde{J}_{\mathcal{M}_{s}}^{1}, \tilde{J}_{\mathcal{M}_{s}}^{2}, . ., \tilde{J}_{\mathcal{M}_{s}}^{2 l-1}, \tilde{J}_{\mathcal{M}_{s}}^{2 l}\right) \leftrightarrow\left(\left(\tilde{J}_{\mathcal{M}_{s}}^{1}, \tilde{J}_{\mathcal{M}_{s}}^{2}\right)_{1-\text { pair }} ; . . ;\left(\tilde{J}_{\mathcal{M}_{s}}^{2 k-1}, \tilde{J}_{\mathcal{M}_{s}}^{2 k}\right)_{k \text {-pair }} ; . . ;\left(\tilde{J}_{\mathcal{M}_{s}}^{2 l-1}, \tilde{J}_{\mathcal{M}_{s}}^{2 l}\right)_{l-\text { pair }}\right)
$$

where,

$$
\tilde{J}_{\mathcal{M}_{s}}^{2 k-1} \stackrel{\text { def }}{=}\left(\frac{\partial \tilde{\mu}_{k}\left(\tau ; \lambda_{k}\right)}{\partial \lambda_{k}}\right)_{\tau} \delta \lambda_{k}, \tilde{J}_{\mathcal{M}_{s}}^{2 k} \stackrel{\text { def }}{=}\left(\frac{\partial \tilde{\sigma}_{k}\left(\tau ; \lambda_{k}\right)}{\partial \lambda_{k}}\right)_{\tau} \delta \lambda_{k}
$$

with $k=1, . ., l$. Similarly, the $2 l$-equations of Jacobi-Levi-Civita can be grouped into $l$-pairs of differential equations with identical structure. Substituting (30), (66) and (64) into (65), after some tedious algebra, the first pair of JLC equations become,

$$
\begin{aligned}
0= & \frac{d^{2} \tilde{J}^{2 k-1}}{d \tau^{2}}+2\left(\Gamma_{12}^{1}\right)^{k} \frac{d \tilde{\sigma}_{k}}{d \tau} \frac{d \tilde{J}^{2 k-1}}{d \tau}+2\left(\Gamma_{12}^{1}\right)^{k} \frac{d \tilde{\mu}_{k}}{d \tau} \frac{d \tilde{J}^{2 k}}{d \tau}+ \\
& +\tilde{J}^{2 k-1}\left[\left(\Gamma_{12}^{1}\right)^{k} \frac{d^{2} \tilde{\sigma}_{k}}{d \tau^{2}}+\left(\partial_{\tilde{\sigma}_{k}}\left(\Gamma_{12}^{1}\right)^{k}+\left(\Gamma_{12}^{1}\right)^{k}\left(\Gamma_{12}^{1}\right)^{k}+\frac{\left(R_{1212}\right)^{k}}{\left(g_{11}\right)^{k}}\right)\left(\frac{d \tilde{\sigma}_{k}}{d \tau}\right)^{2}\right]+ \\
& +\tilde{J}^{2 k}\left[\left(\Gamma_{12}^{1}\right)^{k} \frac{d^{2} \tilde{\mu}_{k}}{d \tau^{2}}+\left(\partial_{\tilde{\sigma}_{k}}\left(\Gamma_{12}^{1}\right)^{k}+\left(\Gamma_{12}^{1}\right)^{k}\left(\Gamma_{12}^{1}\right)^{k}-\frac{\left(R_{1212}\right)^{k}}{\left(g_{11}\right)^{k}}\right) \frac{d \tilde{\mu}_{k}}{d \tau} \frac{d \tilde{\sigma}_{k}}{d \tau}\right],
\end{aligned}
$$

and,

$$
\begin{aligned}
0= & \frac{d^{2} \tilde{J}^{2 k}}{d \tau^{2}}+2\left(\Gamma_{11}^{2}\right)^{k} \frac{d \tilde{\mu}_{k}}{d \tau} \frac{d J^{2 k-1}}{d \tau}+2\left(\Gamma_{22}^{2}\right)^{k} \frac{d \tilde{\sigma}_{k}}{d \tau} \frac{d J^{2 k}}{d \tau}+ \\
& +\tilde{J}^{2 k-1}\left[\left(\Gamma_{11}^{2}\right)^{k} \frac{d^{2} \tilde{\mu}_{k}}{d \tau^{2}}+\left(\partial_{\tilde{\sigma}_{k}}\left(\Gamma_{11}^{2}\right)^{k}+\left(\Gamma_{11}^{2}\right)^{k}\left(\Gamma_{12}^{1}\right)^{k}+\left(\Gamma_{22}^{2}\right)^{k}\left(\Gamma_{11}^{2}\right)^{k}-\frac{\left(R_{1212}\right)^{k}}{\left(g_{22}\right)^{k}}\right) \frac{d \tilde{\mu}_{k}}{d \tau} \frac{d \tilde{\sigma}_{k}}{d \tau}\right]+ \\
& +\tilde{J}^{2}\left[\left(\Gamma_{22}^{2}\right)^{k} \frac{d^{2} \tilde{\sigma}_{k}}{d \tau^{2}}+\left(\partial_{\tilde{\sigma}_{k}}\left(\Gamma_{22}^{2}\right)^{k}+\left(\Gamma_{22}^{2}\right)^{k}\left(\Gamma_{22}^{2}\right)^{k}\right)\left(\frac{d \tilde{\sigma}_{k}}{d \tau}\right)^{2}+\left(\left(\Gamma_{11}^{2}\right)^{k}\left(\Gamma_{21}^{1}\right)^{k}+\frac{\left(R_{1212}\right)^{k}}{\left(g_{22}\right)^{k}}\right)\left(\frac{d \tilde{\mu}_{k}}{d \tau}\right)^{2}\right] .
\end{aligned}
$$

More explicitly, equations (69) and (70) may be written as,

$$
\begin{aligned}
0= & \frac{d^{2} \tilde{J}^{2 k-1}}{d \tau^{2}}-\frac{2}{\tilde{\sigma}_{k}} \frac{d \tilde{\sigma}_{k}}{d \tau} \frac{d \tilde{J}^{2 k-1}}{d \tau}-\frac{2}{\tilde{\sigma}_{k}} \frac{d \tilde{\mu}_{k}}{d \tau} \frac{d \tilde{J}^{2 k}}{d \tau}+\tilde{J}^{2 k-1}\left[-\frac{1}{\tilde{\sigma}_{k}} \frac{d_{k}^{2} \tilde{\sigma}}{d \tau^{2}}+\frac{1}{\tilde{\sigma}_{k}^{2}}\left(\frac{d \tilde{\sigma}_{k}}{d \tau}\right)^{2}\right]+ \\
& +\tilde{J}^{2 k}\left[-\frac{1}{\tilde{\sigma}_{k}} \frac{d^{2} \tilde{\mu}_{k}}{d \tau^{2}}+\frac{3}{\tilde{\sigma}_{k}^{2}} \frac{d \tilde{\mu}_{k}}{d \tau} \frac{d \tilde{\sigma}_{k}}{d \tau}\right],
\end{aligned}
$$

and,

$$
\begin{aligned}
0= & \frac{d^{2} \tilde{J}^{2 k}}{d \tau^{2}}+2 \frac{\alpha_{-}\left(r_{k}\right)}{\alpha_{+}\left(r_{k}\right)} \frac{1}{\tilde{\sigma}_{k}} \frac{d \tilde{\mu}_{k}}{d \tau} \frac{d \tilde{J}^{2 k-1}}{d \tau}-\frac{2}{\tilde{\sigma}_{k}} \frac{d \tilde{\sigma}_{k}}{d \tau} \frac{d \tilde{J}^{2 k}}{d \tau}+\tilde{J}^{2 k-1}\left[\frac{\alpha_{-}\left(r_{k}\right)}{\alpha_{+}\left(r_{k}\right)} \frac{1}{\tilde{\sigma}_{k}} \frac{d^{2} \tilde{\mu}_{k}}{d \tau^{2}}-2 \frac{\alpha_{-}}{\alpha_{+}\left(r_{k}\right)} \frac{1}{\tilde{\sigma}_{k}^{2}} \frac{d \tilde{\mu}_{k}}{d \tau} \frac{d \tilde{\sigma}_{k}}{d \tau}\right]+ \\
& +\tilde{J}^{2 k}\left[-\frac{1}{\tilde{\sigma}_{k}} \frac{d^{2} \tilde{\sigma}_{k}}{d \tau^{2}}+\frac{2}{\tilde{\sigma}_{k}^{2}}\left(\frac{d \tilde{\sigma}_{k}}{d \tau}\right)^{2}-2 \frac{\alpha_{-}\left(r_{k}\right)}{\alpha_{+}\left(r_{k}\right)} \frac{1}{\tilde{\sigma}_{k}^{2}}\left(\frac{d \tilde{\mu}_{k}}{d \tau}\right)^{2}\right] .
\end{aligned}
$$


From (32) and (34), we notice that the asymptotic expansion of $\tilde{\sigma}_{k}$ and the first and second derivative of $\tilde{\mu}_{k}$ and $\tilde{\sigma}_{k}$ are given by,

$$
\begin{aligned}
\tilde{\sigma}_{k}(\tau) & \approx \frac{8 \lambda_{k}^{2}}{\Xi_{k}} \exp \left(-\lambda_{k} \tau\right), \frac{d \tilde{\mu}_{k}}{d \tau} \approx \sqrt{\frac{\alpha_{+}\left(r_{k}\right)}{2 \alpha_{-}\left(r_{k}\right)}} \frac{64 \lambda_{k}^{4}}{\Xi_{k}^{2}} \exp \left(-2 \lambda_{k} \tau\right), \frac{d \tilde{\sigma}_{k}}{d \tau} \approx \frac{8 \lambda_{k}^{3}}{\Xi_{k}} \exp \left(-\lambda_{k} \tau\right), \\
\frac{d^{2} \tilde{\mu}_{k}}{d \tau^{2}} & \approx \sqrt{\frac{\alpha_{+}\left(r_{k}\right)}{2 \alpha_{-}\left(r_{k}\right)}} \frac{128 \lambda_{k}^{5}}{\Xi_{k}^{2}} \exp \left(-2 \lambda_{k} \tau\right), \frac{d^{2} \tilde{\sigma}_{k}}{d \tau^{2}} \approx \frac{8 \lambda_{k}^{4}}{\Xi_{k}} \exp \left(-\lambda_{k} \tau\right) .
\end{aligned}
$$

Substituting (73) into (69) and (70), keeping only the leading terms into the asymptotic expansion, the JLC equations to integrate become,

$$
\frac{d^{2} \tilde{J}^{2 k-1}}{d \tau^{2}}+2 \lambda_{k} \frac{d \tilde{J}^{2 k-1}}{d \tau}-\sqrt{\frac{\alpha_{+}\left(r_{k}\right)}{2 \alpha_{-}\left(r_{k}\right)}} \frac{16 \lambda_{k}^{2}}{\Xi_{k}} \exp \left(-\lambda_{k} \tau\right) \frac{d \tilde{J}^{2 k}}{d \tau}-\sqrt{\frac{\alpha_{+}\left(r_{k}\right)}{2 \alpha_{-}\left(r_{k}\right)}} \frac{8 \lambda_{k}^{3}}{\Xi_{k}} \exp \left(-\lambda_{k} \tau\right) \tilde{J}^{2 k}=0,
$$

and,

$$
\frac{d^{2} \tilde{J}^{2 k}}{d \tau^{2}}+\sqrt{\frac{2 \alpha_{-}\left(r_{k}\right)}{\alpha_{+}\left(r_{k}\right)}} \frac{8 \lambda_{k}^{2}}{\Xi_{k}} \exp \left(-\lambda_{k} \tau\right) \frac{d \tilde{J}^{2 k-1}}{d \tau}+2 \lambda_{k} \frac{d \tilde{J}^{2 k}}{d \tau}+\lambda_{k}^{2} \tilde{J}^{2 k}=0 .
$$

As a working hypothesis, we assume that [36],

$$
\lim _{\tau \rightarrow \infty}\left[\exp \left(-\lambda_{k} \tau\right) \frac{d \tilde{J}^{2 k-1}}{d \tau}\right]=0, \lim _{\tau \rightarrow \infty}\left[\exp \left(-\lambda_{k} \tau\right) \frac{d \tilde{J}^{2 k}}{d \tau}\right]=0, \lim _{\tau \rightarrow \infty}\left[\exp \left(-\lambda_{k} \tau\right) \tilde{J}^{2 k}\right]=0
$$

In order to prove that our assumptions in (76) are correct, we will check a posteriori their consistency. The geodesic deviation equations in (74) and (75) finally become,

$$
\frac{d^{2} \tilde{J}^{2 k-1}}{d \tau^{2}}+2 \lambda_{k} \frac{d \tilde{J}^{2 k-1}}{d \tau}=0, \frac{d^{2} \tilde{J}^{2 k}}{d \tau^{2}}+2 \lambda_{k} \frac{d \tilde{J}^{2 k}}{d \tau}+\lambda_{k}^{2} \tilde{J}^{2 k}=0 .
$$

Integration of (77) leads to the following asymptotic expressions for $\tilde{J}_{\mathcal{M}_{s}}^{2 k-1}(\tau)$ and $\tilde{J}_{\mathcal{M}_{s}}^{2 k}(\tau)$,

$$
\tilde{J}_{\mathcal{M}_{s}}^{2 k-1}(\tau)=C_{0}^{(k)}+C_{1}^{(k)} \exp \left(-2 \lambda_{k} \tau\right), \tilde{J}_{\mathcal{M}_{s}}^{2 k}(\tau)=C_{2}^{(k)} \exp \left(-\lambda_{k} \tau\right)+C_{3}^{(k)} \tau \exp \left(-2 \lambda_{k} \tau\right),
$$

where $C_{w}^{(k)}$ are real constant of integration with $w=1, . ., 3$. Notice that conditions (76) are satisfied and therefore our assumption are compatible with the solutions obtained.

Consider the Jacobi vector field components $\left\{\tilde{J}^{\mu}\right\}_{\mu=1, . ., l}$ defined in (67) and its magnitude $\tilde{J}_{\mathcal{M}_{s}}$,

$$
\tilde{J}_{\mathcal{M}_{s}}^{2} \stackrel{\text { def }}{=}\left(\tilde{J}_{\mathcal{M}_{s}}\right)^{\mu}\left(\tilde{J}_{\mathcal{M}_{s}}\right)_{\mu}
$$

The magnitude $\tilde{J}_{\mathcal{M}_{s}}$ is called the Jacobi field intensity. Using (28), Equation (79) becomes,

$$
\tilde{J}_{\mathcal{M}_{s}}^{2}(\tau) \stackrel{\text { def }}{=} \sum_{k=1}^{l}\left\{\frac{\alpha_{-}\left(r_{k}\right)}{\left[a_{1}\left(r_{k}\right)\right]^{2}} \frac{1}{\tilde{\sigma}_{k}^{2}}\left[\tilde{J}_{\mathcal{M}_{s}}^{2 k-1}(\tau)\right]^{2}+\frac{\alpha_{+}\left(r_{k}\right)}{\left[a_{1}\left(r_{k}\right)\right]^{2}} \frac{1}{\tilde{\sigma}_{k}^{2}}\left[\tilde{J}_{\mathcal{M}_{s}}^{2 k}(\tau)\right]^{2}\right\}
$$

Substituting (173) and (78) into (80), keeping only the leading terms in the asymptotic expansion of $\tilde{J}_{\mathcal{M}_{s}}^{2}(\tau)$, we obtain

$$
\tilde{J}_{\mathcal{M}_{s}}^{2}(\tau) \stackrel{\tau \rightarrow \infty}{\approx} \sum_{k=1}^{l}\left[\frac{\alpha_{-}\left(r_{k}\right)}{\left[a_{1}\left(r_{k}\right)\right]^{2}}\left(\frac{C_{0}^{(k)} \Xi_{k}}{8 \lambda_{k}^{2}}\right)^{2} \exp \left(2 \lambda_{k} \tau\right)\right] .
$$

Let us rewrite the quantity $\tilde{J}_{\mathcal{M}_{s}}^{2}(\tau)$ in terms of "attenuation factors" $\mathcal{A}\left(r_{k}\right)$ and elementary quadratic Jacobi vector field components $j_{\mathcal{M}_{s}}^{2}\left(\tau ; \lambda_{k}\right)$ given by,

$$
\widetilde{\mathcal{A}}_{k}\left(r_{k}\right) \stackrel{\text { def }}{=} \frac{\alpha_{-}\left(r_{k}\right)}{\left[a_{1}\left(r_{k}\right)\right]^{2}}=\frac{2 r_{k}\left(3-\sqrt{1+4 r_{k}^{2}}\right)}{\left(1+\sqrt{1+4 r_{k}^{2}}\right)^{2}},
$$


and,

$$
\tilde{j}_{\mathcal{M}_{s}}^{2}\left(\tau ; \lambda_{k}\right) \stackrel{\text { def }}{=}\left(\frac{C_{0}^{(k)} \Xi_{k}}{8 \lambda_{k}^{2}}\right)^{2} \exp \left(2 \lambda_{k} \tau\right),
$$

respectively. Notice that $\widetilde{\mathcal{A}}_{k}\left(r_{k}\right)$ is a bounded function of the correlation coefficient $r_{k} \in(0,1)$ and its maximum is reached for $\bar{r}_{k}=\sqrt{2-\sqrt{2}} \simeq 0.77$,

$$
\widetilde{\mathcal{A}}_{k \max }\left(r_{k}\right) \stackrel{\text { def }}{=} \max _{r_{k} \in(0,1)} \widetilde{\mathcal{A}_{k}}\left(r_{k}\right)=\widetilde{\mathcal{A}}_{k}\left(\bar{r}_{k}\right)=3-2 \sqrt{2} \simeq 0.17
$$

Therefore, substituting (82) and (831) into (81) and considering the boundedness of the attenuation factors in (84), the square of the Jacobi field intensity $\tilde{J}_{\mathcal{M}_{s}}^{2}(\tau)$ may be written as,

$$
\tilde{J}_{\mathcal{M}_{s}}^{2}\left(\tau ; \lambda_{1}, . ., \lambda_{l}\right) \stackrel{\tau \rightarrow \infty}{\approx} \sum_{k=1}^{l} \widetilde{\mathcal{A}}_{k}\left(r_{k}\right) \tilde{j}_{\mathcal{M}_{s}}^{2}\left(\tau ; \lambda_{k}\right)
$$

Let us now consider the asymptotic behavior of $J_{\mathcal{M}_{s}}$,

$$
J_{\mathcal{M}_{s}}^{2} \stackrel{\text { def }}{=} \sum_{k=1}^{l}\left\{\frac{1}{\sigma_{k}^{2}}\left[J_{\mathcal{M}_{s}}^{(2 k-1)}\right]^{2}+\frac{2 r_{k}}{\sigma_{k}^{2}} J_{\mathcal{M}_{s}}^{(2 k-1)} J_{\mathcal{M}_{s}}^{(2 k)}+\frac{2}{\sigma_{k}^{2}}\left[J_{\mathcal{M}_{s}}^{(2 k)}\right]^{2}\right\}
$$

From (23) and (62), it follows that the Jacobi field components $\left(J_{\mathcal{M}_{s}}^{(2 k-1)}, J_{\mathcal{M}_{s}}^{(2 k)}\right)$ with $k=1, . ., l$ are related to the Jacobi field components $\left(\tilde{J}_{\mathcal{M}_{s}}^{(2 k-1)}, \tilde{J}_{\mathcal{M}_{s}}^{(2 k)}\right)$ in the following way,

$$
J_{\mathcal{M}_{s}}^{(2 k-1)} \stackrel{\text { def }}{=} \tilde{J}_{\mathcal{M}_{s}}^{(2 k-1)}+\tilde{J}_{\mathcal{M}_{s}}^{(2 k)}
$$

and,

$$
J_{\mathcal{M}_{s}}^{(2 k)} \stackrel{\text { def }}{=} \frac{1-\sqrt{\Delta\left(r_{k}\right)}}{2 r_{k}} \tilde{J}_{\mathcal{M}_{s}}^{(2 k-1)}+\frac{1+\sqrt{\Delta\left(r_{k}\right)}}{2 r_{k}} \tilde{J}_{\mathcal{M}_{s}}^{(2 k)}
$$

Considering (78) and substituting (87) and (88) into (86), the asymptotic behavior of $J_{\mathcal{M}_{s}}^{2}$ is given by,

$$
J_{\mathcal{M}_{s}}^{2} \approx \sum_{k=1}^{l}\left\{\left[1+2 r_{k} \frac{1-\sqrt{\Delta\left(r_{k}\right)}}{2 r_{k}}+2\left(\frac{1-\sqrt{\Delta\left(r_{k}\right)}}{2 r_{k}}\right)^{2}\right] \frac{1}{\sigma_{k}^{2}}\left[\tilde{J}_{\mathcal{M}_{s}}^{(2 k-1)}\right]^{2}\right\} .
$$

Substituting (37) and (78) into (89) and keeping only the leading terms into the asymptotic expansion, we obtain,

$$
\left(J_{\mathcal{M}_{s}}^{2}\right)_{\text {embedded }}\left(\tau ; \lambda_{1}, . ., \lambda_{l}\right) \approx \sum_{k=1}^{l} \mathcal{A}_{k}\left(r_{k}\right) j_{\mathcal{M}_{s}}^{2}\left(\tau ; \lambda_{k}\right),
$$

where $j_{\mathcal{M}_{s}}^{2}\left(\tau ; \lambda_{k}\right)=\tilde{j}_{\mathcal{M}_{s}}^{2}\left(\tau ; \lambda_{k}\right)$ is defined in (83) and the new attenuation function is given by,

$$
\mathcal{A}_{k}\left(r_{k}\right) \stackrel{\text { def }}{=} \frac{4 r_{k}^{2}\left[1+2 r_{k} a_{0}\left(r_{k}\right)+a_{0}^{2}\left(r_{k}\right)\right]}{\left[1+a_{1}\left(r_{k}\right)\right]^{2}}
$$

where $a_{0}\left(r_{k}\right)$ and $a_{1}\left(r_{k}\right)$ are defined in (25). Notice that $\mathcal{A}_{k}\left(r_{k}\right)$ is a bounded function of the correlation coefficient $r_{k} \in(0,1)$ and its maximum is reached for $\bar{r}_{k} \simeq 0.65$,

$$
\mathcal{A}_{k \max }\left(r_{k}\right) \stackrel{\text { def }}{=} \max _{r_{k} \in(0,1)} \mathcal{A}_{k}\left(r_{k}\right)=\mathcal{A}_{k}\left(\bar{r}_{k}\right) \simeq 0.15
$$

In [36, 44], it was shown that in absence of constraints, the model here considered leads to an asymptotic behavior of the Jacobi fields given by,

$$
\left(J_{\mathcal{M}_{s}}^{2}\right)_{\text {larger }}\left(\tau ; \lambda_{1}, . ., \lambda_{2 l}\right) \stackrel{\tau \rightarrow \infty}{\approx} \sum_{k=1}^{2 l} j_{\mathcal{M}_{s}}^{2}\left(\tau ; \lambda_{k}\right)
$$


Therefore, from equations (93) and (90), we obtain,

$$
0 \leq\left(\frac{\left[\left(J_{\mathcal{M}_{s}}^{2}\right)_{\text {embedded }}\left(\tau ; \lambda_{1}, . ., \lambda_{l}\right)\right]_{\bar{k}}}{\left[\left(J_{\mathcal{M}_{s}}^{2}\right)_{\text {larger }}\left(\tau ; \lambda_{1}, . ., \lambda_{l}\right)\right]_{\bar{k}}}\right)^{\frac{1}{2}} \approx \sqrt{\frac{4 r_{\bar{k}}^{2}\left[1+2 r_{\bar{k}} a_{0}\left(r_{\bar{k}}\right)+a_{0}^{2}\left(r_{\bar{k}}\right)\right]}{\left[1+a_{1}\left(r_{\bar{k}}\right)\right]^{2}}} \lesssim 0.4<1,
$$

We conclude that the appearance of embedding constraints among the Gaussian statistical macrovariables on the larger 4l-dimensional curved manifold leads to an attenuation of the asymptotic exponential divergence of the Jacobi field intensity on the embedded $2 l$-dimensional manifold. This is a quantitative indication that the information geometric complexity of a system decreases in the presence of emerging correlational structures.

\section{FINAL REMARKS}

In this article, we characterized the complexity of geodesic paths on a curved statistical manifold $\mathcal{M}_{s}$ through the asymptotic computation of the information geometric complexity $\mathcal{V}_{\mathcal{M}_{s}}$ and the Jacobi vector field intensity $J_{\mathcal{M}}$. We considered a manifold a $2 l$-dimensional Gaussian model $\mathcal{M}_{s}$ reproduced by an appropriate embedding in a larger $4 l$-dimensional Gaussian manifold and endowed with a Fisher-Rao information metric $g_{\mu \nu}(\Theta)$ with non-trivial off diagonal terms. Such terms in the information metric on the embedded manifold emerged due to the presence of a correlational structure (embedding constraints) among the statistical variables on the larger manifold and were characterized by macroscopic correlational coefficients $r_{k}$. First, we observed a power law decay of the information geometric complexity at a rate determined by the coefficients $r_{k}$ and concluded that the non-trivial off diagonal terms lead to the emergence of an asymptotic information geometric compression of the explored macrostates $\Theta$ on $\mathcal{M}_{s}$. Finally, we observed that the presence of such embedding constraints lead to an attenuation of the asymptotic exponential divergence of the Jacobi vector field intensity.

The relevance of such results becomes evident when compared to what happens in the larger Gaussian statistical model (absence of constraints) [36, 44]. In such case, the information geometric entropy of the 4l-dimensional (larger and uncorrelated) Gaussian model increases linearly in time and its complexity diverges exponentially at a rate determined by $\lambda_{k}$, the Lyapunov exponents of the statistical trajectories of the system [25] and the Jacobi field intensity diverges exponentially without any attenuation factor. It seems that our measure of complexity not only can quantify the degree of chaoticity of a physical system, but it also adequately captures the correlational structure (relationship between system's components, 3, 9-11]) in its behavior. In the model studied, the emergence of structure appears in terms of non-trivial off diagonal elements in the Fisher-Rao information metric. Such structure leads to the information geometric compression of $\widetilde{v o l}\left[\mathcal{D}_{\Theta}^{\text {(geodesic) }}(\tau)\right]$ and, thus, to a reduction of the complexity of the path leading to $\Theta_{\text {final }}$ from $\Theta_{\text {initial }}$.

Information Geometry and Maximum (relative) Entropy methods hold great promise for solving computational problems of interest in classical and quantum physics in terms of their probabilistic description on curved statistical manifolds. Our theoretical formalism allows us to tackle physics problems through statistical inference and information geometric techniques, that is Riemannian geometric techniques applied to probability theory. The macroscopic behavior of an arbitrary complex system is a consequence of the underlying statistical structure of the microscopic degrees of freedom of the system being considered.

As a side remark, we point out two more facts: 1) Probabilistic concepts are naturally incorporated into the fundamentally statistical quantum theory. Furthermore, describing and understanding the complexity of quantum motion is still an open problem since our present knowledge on the relations among complexity, chaoticity and dynamical stochasticity are not satisfactory at all [5]; 2) Riemannian geometric tools are currently being used to characterize the quantum gate complexity in quantum computing [50 53]. In [50], the problem of finding quantum circuits was recasted as a geometric problem. It was shown that finding optimal quantum circuits is essentially equivalent to finding the shortest path (geodesic) between two points in a certain curved geometry. In light of these two considerations and in view of the results obtained thus far, we are confident the work presented here constitutes a further important step towards the characterization of the dynamical complexity of microscopically correlated multidimensional Gaussian statistical models, and other models of relevance in more realistic physical systems. We hope to extend this approach in the field of Quantum Information to better understand the connection between quantum entanglement and quantum complexity [5, 41, 42, 54, 55]. 


\section{Acknowledgments}

C. C. thanks Sean Alan Ali, Adom Giffin, Akira Inomata, John Kimball, Kevin Knuth and Carlos Rodriguez for useful discussions on chaos and complexity. C. C. is especially indebted to Ariel Caticha for important advises and illuminating comments. This work was supported by the European Community's Seventh Framework Program FP7/2007-2013 under grant agreement 213681 (CORNER Project).

\section{Appendix A: Derivation of the line element}

We derive Equation (6). For the sake of clarity, we consider a two-dimensional Gaussian probability distribution $p\left(x_{1}, x_{2} \mid \mu_{1}, \mu_{2}, \sigma_{1}\right)$ obtained from the Gaussian distribution $p\left(x_{1}, x_{2} \mid \mu_{1}, \mu_{2}, \sigma_{1}, \sigma_{2}\right)$ setting $\sigma_{1}=\sigma_{2}$ and defined as,

$$
p\left(x_{1}, x_{2} \mid \mu_{1}, \mu_{2}, \sigma_{1}\right)=\frac{1}{2 \pi \sigma_{1}^{2}} \exp \left[-\frac{1}{2 \sigma_{1}^{2}}\left[\left(x_{1}-\mu_{1}\right)^{2}+\left(x_{2}-\mu_{2}\right)^{2}\right]\right] .
$$

The Fisher-Rao information metric in the three-dimensional statistical manifold $\left(\mu_{1}, \mu_{2}, \sigma_{1}\right)$ is given by,

$$
d s^{2}=\frac{1}{\sigma_{1}^{2}}\left(d \mu_{1}^{2}+d \mu_{2}^{2}+4 d \sigma_{1}^{2}\right) .
$$

Consider the two-dimensional submanifold embedded as a slice in the three-dimensional space defined by the following embedding constraint,

$$
\mu_{2}=\mu_{2}\left(\mu_{1}, \sigma_{1}\right) .
$$

The Gaussian distributions $p\left(x_{1}, x_{2} \mid \mu_{1}, \mu_{2}\left(\mu_{1}, \sigma_{1}\right), \sigma_{1}\right) \equiv \tilde{p}\left(x_{1}, x_{2} \mid \mu_{1}, \sigma_{1}\right)$ belonging to this submanifold are such that $\left\langle x_{2}\right\rangle=\mu_{2}$ and $\left\langle x_{1}\right\rangle=\mu_{1}$ are not independent, they are related in a peculiar way. From (A3), we obtain

$$
d \mu_{2}=\frac{\partial \mu_{2}}{\partial \mu_{1}} d \mu_{1}+\frac{\partial \mu_{2}}{\partial \sigma_{1}} d \sigma_{1}
$$

that is,

$$
d \mu_{2}^{2}=\left(\frac{\partial \mu_{2}}{\partial \mu_{1}}\right)^{2} d \mu_{1}^{2}+\left(\frac{\partial \mu_{2}}{\partial \sigma_{1}}\right)^{2} d \sigma_{1}^{2}+2 \frac{\partial \mu_{2}}{\partial \mu_{1}} \frac{\partial \mu_{2}}{\partial \sigma_{1}} d \mu_{1} d \sigma_{1}
$$

Substituting (A5) in (A2), the information metric becomes,

$$
d s^{2}=\frac{1}{\sigma_{1}^{2}}\left[A_{\mu_{1} \mu_{1}} d \mu_{1}^{2}+2 A_{\mu_{1} \sigma_{1}} d \mu_{1} d \sigma_{1}+2 A_{\sigma_{1} \sigma_{1}} d \sigma_{1}^{2}\right]
$$

where the coefficients $A_{\mu_{1} \mu_{1}}, A_{\mu_{1} \sigma_{1}}$ and $A_{\sigma_{1} \sigma_{1}}$ are given by,

$$
A_{\mu_{1} \mu_{1}} \stackrel{\text { def }}{=} 1+\left(\frac{\partial \mu_{2}}{\partial \mu_{1}}\right)^{2}, A_{\mu_{1} \sigma_{1}} \stackrel{\text { def }}{=} \frac{\partial \mu_{2}}{\partial \mu_{1}} \frac{\partial \mu_{2}}{\partial \sigma_{1}} \text { and, } A_{\sigma_{1} \sigma_{1}} \stackrel{\text { def }}{=} 2+\frac{1}{2}\left(\frac{\partial \mu_{2}}{\partial \sigma_{1}}\right)^{2}
$$

Re-scaling the variables in such a way that $\tilde{\mu}_{1} \stackrel{\text { def }}{=} A_{\mu_{1} \mu_{1}}^{\frac{1}{2}} \mu_{1}$ and $\tilde{\sigma}_{1} \stackrel{\text { def }}{=} A_{\sigma_{1} \sigma_{1}}^{\frac{1}{2}} \sigma_{1}$ and assuming that the coefficients $A_{\mu_{1} \mu_{1}}, A_{\mu_{1} \sigma_{1}}$ and $A_{\sigma_{1} \sigma_{1}}$ are constants, the line element in (A6) becomes

$$
d s^{2}=A_{\sigma_{1} \sigma_{1}} \frac{1}{\tilde{\sigma}_{1}^{2}}\left[d \tilde{\mu}_{1}^{2}+2 r_{1} d \tilde{\mu}_{1} d \tilde{\sigma}_{1}+2 d \tilde{\sigma}_{1}^{2}\right]
$$

with,

$$
r_{1} \stackrel{\text { def }}{=} \frac{A_{\mu_{1} \sigma_{1}}}{A_{\mu_{1} \mu_{1}}^{\frac{1}{2}} A_{\sigma_{1} \sigma_{1}}^{\frac{1}{2}}}=\frac{\frac{\partial \mu_{2}}{\partial \mu_{1}} \frac{\partial \mu_{2}}{\partial \sigma_{1}}}{\left[1+\left(\frac{\partial \mu_{2}}{\partial \mu_{1}}\right)^{2}\right]^{\frac{1}{2}}\left[2+\frac{1}{2}\left(\frac{\partial \mu_{2}}{\partial \sigma_{1}}\right)^{2}\right]^{\frac{1}{2}}}
$$


From (A7), it follows that the embedding defining the two-dimensional submanifold in the larger three-dimensional manifold of Gaussians parametrized by $\left(\mu_{1}, \mu_{2}, \sigma_{1}\right)$ is given by,

$$
\mu_{2}\left(\mu_{1}, \sigma_{1}\right)=a_{1}^{(1)} \mu_{1}+a_{2}^{(1)} \sigma_{1}=\frac{a_{1}^{(1)}}{\sqrt{1+\left[a_{1}^{(1)}\right]^{2}}} \tilde{\mu}_{1}+\frac{a_{2}^{(1)}}{\sqrt{2+\frac{1}{2}\left[a_{2}^{(1)}\right]^{2}}} \tilde{\sigma}_{1},
$$

with,

$$
a_{1}^{(1)} \stackrel{\text { def }}{=}\left(A_{\mu_{1} \mu_{1}}-1\right)^{\frac{1}{2}} \text { and, } a_{2}^{(1)} \stackrel{\text { def }}{=}\left(2 A_{\sigma_{1} \sigma_{1}}-4\right)^{\frac{1}{2}} .
$$

Finally, using (A10) and (A9), the explicit expression for $r_{1}$ becomes

$$
r_{1}=\frac{a_{1}^{(1)} a_{2}^{(1)}}{\sqrt{1+\left[a_{1}^{(1)}\right]^{2}} \sqrt{2+\frac{1}{2}\left[a_{2}^{(1)}\right]^{2}}} .
$$

From (A12), it is transparent that the explicit expression for $r_{1}$ depends on the functional parametric form of the embedding constraint in (A10).

[1] R. Landauer, " A simple measure of complexity", Nature 336, 306 (1988).

[2] M. Gell-Mann, "What is Complexity?", Complexity 1, 1 (1995).

[3] D. P. Feldman and J. P. Crutchfield, "Measures of statistical complexity: Why?", Phys. Lett. A238, 244 (1998).

[4] M. Ohya, "Complexities and Their Applications to Characterization of Chaos", Int. J. Theor. Phys. 37, 495 (1998).

[5] G. Benenti and G. Casati, "How complex is quantum motion?", Phys. Rev E79, 025201 (2009).

[6] S. Wolfram, "Universality and Complexity in Cellular Automata", Physica D10, 1 (1984).

[7] S. Wolfram, "Origin of Randomness in Physical Systems", Phys. Rev. Lett. 55, 449 (1985).

[8] M. Rasetti, "Uncertainty, Predictability and Decidability in Chaotic Dynamical Systems", Chaos, Solitons \& Fractals 5, 133 (1995).

[9] B. A. Huberman and T. Hogg, "Complexity and Adaption", Physica D22, 376 (1986).

[10] P. Grassberger, "Toward a Quantitative Theory of Self-Generated Complexity", Int. J. Theor. Phys. 25, 907 (1986).

[11] J. P. Crutchfield and K. Young, "Inferring Statistical Complexity", Phys. Rev. Lett. 63, 105 (1989).

[12] A. N. Kolmogorov, "Three approaches to the quantitative definition of information", Probl. Inf. Transm. (USSR) 1, 4 (1965); "Logical basis for information theory and probability theory", IEEE Trans. Inf. Theory, IT14, 662 (1968).

[13] Y. Pesin, "Characteristic Lyapunov exponents and smooth ergodic theory", English transl., Russian Math. Surveys 32, 55 (1977).

[14] A. A. Brudno, "Entropy and the complexity of trajectories of a dynamical system", Trans. Moscow Math. Soc. 2, 127 (1983).

[15] F. Blume, "On the relation between entropy and the average complexity of trajectories in dynamical systems", Comp. Complex. 9, 146 (2000).

[16] P. Szepfalusy, "Characterization of Chaos and Complexity by Properties of Dynamical Entropies", Physica Scripta T25, 226 (1989).

[17] A. Wehrl, "General properties of entropy", Rev. Mod. Phys. 50, 221 (1978).

[18] C. H. Bennett, "How to Define Complexity in Physics, and Why", in "Complexity, Entropy and the Physics of Information", SFI Studies in the Sciences of Complexity, vol.VIII, Ed. W. H. Zurek, Addison-Wesley (1990).

[19] S. Lloyd and H. Pagels, "Complexity as Thermodynamic Depth", Annals of Physics 188, 186 (1988).

[20] C. M. Papadimitriou, "Computational Complexity", Addison-Wesley, Massachusetts (1994).

[21] J. Rissanen, "Stochastic complexity and modeling", Ann. Stat. 14, 1080 (1986).

[22] V. M. Alekseev and M. V. Yacobson, "Symbolic dynamics and hyperbolic dynamic systems", Phys. Rep. 75, 287 (1982).

[23] M. A. Nielsen and I. L. Chuang, "Quantum Computation and Information", Cambridge University Press (2000).

[24] A. Caticha, "Entropic Dynamics", in Bayesian Inference and Maximum Entropy Methods in Science and Engineering, ed. by R.L. Fry, AIP Conf. Proc. 617, 302 (2002).

[25] C. Cafaro, "The Information Geometry of Chaos", Ph. D. Thesis, State University of New York at Albany, USA (2008).

[26] C. Cafaro, "Works on an information geometrodynamical approach to chaos", Chaos, Solitons \& Fractals 41, 886 (2009).

[27] A. Caticha and R. Preuss, "Maximum entropy and Bayesian data analysis: Entropic prior distributions", Phys. Rev. E70, 046127 (2004).

[28] A. Caticha, "Relative Entropy and Inductive Inference", Bayesian Inference and Maximum Entropy Methods in Science and Engineering,ed. by G. Erickson and Y. Zhai, AIP Conf. Proc. 707, 75 (2004). 
[29] A. Caticha and A. Giffin, "Updating Probabilities", in Bayesian Inference and Maximum Entropy Methods in Science and Engineering, ed. by Ali Mohammad-Djafari, AIP Conf. Proc. 872, 31 (2006).

[30] A. Giffin, "Maximum Entropy: The Universal Method for Inference", Ph. D. Thesis, State University of New York at Albany, USA (2008).

[31] S. Amari and H. Nagaoka, Methods of Information Geometry, American Mathematical Society, Oxford University Press, 2000.

[32] L. Casetti et al. , "Riemannian theory of Hamiltonian chaos and Lyapunov exponents", Phys. Rev. E54, 5969 (1996).

[33] M. Di Bari and P. Cipriani, " Geometry and Chaos on Riemann and Finsler Manifolds", Planet. Space Sci. 46, 1543 (1998).

[34] C. G. J. Jacobi, "Vorlesungen uber Dynamik", Reimer, Berlin (1866).

[35] T. Kawabe, "Indicator of chaos based on the Riemannian geometric approach", Phys. Rev. E71, 017201 (2005); T. Kawabe, "Chaos based on Riemannian geometric approach to Abelian-Higgs dynamical system", Phys. Rev. E67, 016201 (2003).

[36] C. Cafaro and S. A. Ali, "Jacobi Fields on Statistical Manifolds of Negative Curvature", Physica D234, 70 (2007).

[37] M. Tribus, "Rational Descriptions, Decisions and Designs", Pergamon Press (1969).

[38] A. Caticha and C. Cafaro, "From Information Geometry to Newtonian Dynamics", in Bayesian Inference and Maximum Entropy Methods in Science and Engineering, ed. by K. Knuth et al., AIP Conf. Proc. 954, 165 (2007).

[39] S. A. Ali, C. Cafaro, D.-H. Kim, S. Mancini, "The Effect Of Microscopic Correlations On The Information Geometric Complexity Of Gaussian Statistical Models", Physica A389, 3117 (2010).

[40] C. Cafaro and S. A. Ali, "Geometrodynamics of Information on Curved Statistical Manifolds and its Applications to Chaos", EJTP 5, 139 (2008).

[41] C. Cafaro, "Information geometry, inference methods and chaotic energy levels statistics", Mod. Phys. Lett. B22, 1879 (2008).

[42] C. Cafaro and S. A. Ali, "Can chaotic quantum energy levels statistics be characterized using information geometry and inference methods?", Physica A387, 6876 (2008).

[43] C. Cafaro and S. Mancini, "On the Complexity of Statistical Models Admitting Correlations", arXiv: math-ph/0905.2907 (2009).

[44] C. Cafaro, "Information-Geometric Indicators of Chaos in Gaussian Models on Statistical Manifolds of Negative Ricci Curvature", Int. J. Theor. Phys. 47, 2924 (2008).

[45] J. M. Lee, "Riemannian Manifolds: An Introduction to Curvature", Springer-Verlag (1997).

[46] G. Casati and B. V. Chirikov, "The legacy of chaos in quantum mechanics", in Quantum Chaos: Between Order and Disorder, edited by G. Casati and B. V. Chirikov, Cambridge University Press (1995).

[47] F. De Felice and J. S. Clarke, "Relativity on Curved Manifolds", Cambridge University Press (1990).

[48] H. C. Ohanian and R. Ruffini, "Gravitation and Spacetime", W.W. Norton \& Company (1994).

[49] C. Chicone and B. Mashhoon," The generalized Jacobi equation", Class. Quantum Grav. 19, 4231 (2002).

[50] M. A. Nielsen et. al., "Quantum Computation as Geometry", Science 311, 1133 (2006).

[51] M. R. Dowling and M. A. Nielsen, "The Geometry of Quantum Computation", Quantum Information \& Computation 8, 0861 (2008).

[52] H. E. Brandt, "Riemannian Geometry of Quantum Computation", Nonlinear Analysis 71, 474 (2009).

[53] H. E. Brandt, "Riemannian curvature in the differential geometry of quantum computation", Physica E42, 449 (2010).

[54] M. A. Nielsen, "Quantum information science as an approach to complex quantum systems", arXiv:quant-ph/0208078 (2002).

[55] T. Prosen, " Chaos and Complexity of Quantum Motion", J. Phys. A40, 7881 (2007). 